\title{
Der Versuch einer großen Integration
}

\author{
Paul Martini und der erste Nachkriegskongress der Deutschen \\ Gesellschaft für Innere Medizin
}

\author{
Ralf Forsbach und Hans-Georg Hofer
}

\begin{abstract}
Attempting a Great Integration. Paul Martini and the First Post-War Conference of the German Society for Internal Medicine
\end{abstract}

\begin{abstract}
The long established German Society for Internal Medicine (DGIM) profoundly incriminated itself through its actions and positions during the National Socialist era. The German clinical physician Paul Martini assumed the part of reorganizing the DGIM prior to its first post-war convention in 1948 in Karlsruhe. Martini, who himself had opposed the Nazi regime, adopted a course of comprehensive integration. He strived to incorporate both physicians who had been persecuted by the Nazi Regime as well as former moderate National Socialists into the DGIM. At the same time he campaigned to preserve the pan-German nature of the conferences and aimed rapidly to make the DGIM re-compatible with international research. However, this path led to an allegedly apolitical focus on science and decades of largely failing to confront its Nazi past.
\end{abstract}

Keywords: Contemporary history of medicine, Paul Martini, Internal medicine, National Socialism, Post-war era, Occupation period

\begin{abstract}
Die traditionsreiche Deutsche Gesellschaft für Innere Medizin (DGIM) war durch ihr Agieren in der NS-Zeit belastet. Vor ihrem ersten Nachkriegskongress 1948 in Karlsruhe fiel dem Bonner Klinikdirektor Paul Martini die Rolle eines Reorganisators zu. Martini, der während der NS-Zeit in Opposition zum Regime gestanden hatte, wählte einen Kurs umfassender Integration. Verfolgte des NS-Staats versuchte er ebenso in die DGIM einzubinden wie einstige gemäßigte Nationalsozialisten. Zugleich bemühte er sich um die Bewahrung des gesamtdeutschen Charakters der Kongresse sowie um einen zügigen Anschluss an die internationale Forschung. Dennoch führte der eingeschlagene Weg die DGIM in nur scheinbar unpolitische Wissenschaftlichkeit und Jahrzehnte weitgehender Kritiklosigkeit gegenüber ihrer NS-Geschichte.
\end{abstract}

Schlüsselwörter: Zeitgeschichte der Medizin, Paul Martini, Innere Medizin, Nationalsozialismus, Nachkriegszeit, Besatzungszeit

Medizinische Fachgesellschaften sind in den vergangenen Jahren verstärkt in das Blickfeld der historischen Forschung geraten, oft unter besonderer Berücksichtigung der NS-Zeit und der frühen Jahre der Bundesrepublik. ${ }^{1}$ Diese Entwicklung stand im Einklang mit dem Bemühen, das Verhalten von Forschern, Funktionären und Politikern innerhalb naturwissenschaftlicher und naturwissenschaftsnaher Fächer in der NS-Zeit klarer zu konturieren 
sowie Strukturen und Netzwerke offenzulegen (Maier 2015; Deichmann 1995; 2001; Epple \& Bergmann 2008). Weniger intensiv betrachtet wurde bislang die Besatzungszeit, in der sich die Fächer mit ihren Fachgesellschaften unter völlig veränderten Bedingungen neu konstituierten. Der Umgang mit den in der NS-Zeit Exponierten spielte ebenso eine Rolle wie die politische und - noch nachgeordnet - wissenschaftliche Ausrichtung. Dabei erschwerten die angespannte wirtschaftliche Lage, aber auch die durch die Besatzungsmächte eingeschränkten Reisemöglichkeiten die Kommunikation der Entscheidungsträger.

Die schwierigen Lebensbedingungen der Nachkriegsjahre erklären die häufig schlechte Quellenlage. So beschränken sich die hauseigenen Bestände der internistischen Fachgesellschaft im Wesentlichen auf wenige Protokolle. Umso wertvoller ist die erhalten gebliebene Korrespondenz des Bonner Direktors der Medizinischen Klinik, Paul Martini (1889-1964), die sich mit der Planung und Durchführung der ersten Tagung der Deutschen Gesellschaft für Innere Medizin (DGIM) im Mai 1948 befasst. Hierin werden die nicht zuletzt durch die politische Vergangenheit, vor allem aber auch durch die Bedingungen der alliierten Besatzung gegebenen Einflüsse auf die Neukonstituierung der DGIM deutlich.

Der Nachlass von Paul Martini ermöglicht zudem eine genaue Rekonstruktion der Interessen und Handlungen jener Akteure, die - zum Teil in offener Rivalität - um die Neuausrichtung der Gesellschaft rangen. Die Suche nach einem geeigneten Ort zur Abhaltung des Kongresses war der äußere Anlass für brieflich ausgetragene Kontroversen um die fachpolitische und inhaltliche Ausrichtung der Gesellschaft. Dabei wurden die zur Auswahl stehenden Städte auf ihre Symbolkraft für eine Teilung Deutschlands und der DGIM beziehungsweise im Umkehrschluss für den erklärten Willen zum Erhalt der Einheit gedeutet. Maßgebliche Diskutanten waren neben Martini Theodor Brugsch (1878-1963) in Berlin (Ost) und der Senior der DGIM, Ludolph Brauer (1865-1951), in München. Erkennbar wird in diesem Zusammenhang auch, wie aus der NS-Zeit Belastete sich und ihr Umfeld wahrnahmen - zu einer Zeit, als die Bemühungen um „Entnazifizierung“ und „(Selbst)Reinigung“ der Universitätsmedizin oft noch im Gange waren (Ferdinand et al. 2013; Oehler-Klein \& Roelcke 2007). Damit korrespondiert die Frage nach dem Umgang mit den vertriebenen, teilweise aus der Emigration zurückkehrenden Mitgliedern. Eine erste Antwort darauf geben die vom DGIM-Vorstand ausgesprochenen Einladungen und Ehrungen.

Die 1882 als „Congress für Innere Medizin“ in Wiesbaden gegründete Deutsche Gesellschaft für Innere Medizin zählt bis heute zu den ältesten, größten und wichtigsten medizinischen Fachgesellschaften. 1933 hatte sie sich rasch den neuen Machthabern angepasst. Der gewählte Vorsitzen- 
de Leopold Lichtwitz (1876-1943), Direktor des Berliner Rudolf-VirchowKrankenhauses, wurde abgesetzt und als Jude verfolgt; am 30. März 1933 verließ er Deutschland. Sein Nachfolger, der zunächst in Kiel, dann in München lehrende Alfred Schittenhelm (1874-1954), etablierte den Kurs der Selbstgleichschaltung. Auch die anderen, im Jahresturnus wechselnden DGIM-Vorsitzenden von 1935 bis 1940 vermieden Konfrontationen mit dem Regime, waren zum Teil sogar an NS-Verbrechen beteiligt. Die Tradition der jährlichen Wiesbadener Kongresse der DGIM brach 1940 ab. Der für das Folgejahr gewählte Wiener Klinikdirektor Hans Eppinger (1879-1946) konnte keinen regulären Kongress durchführen. Er blieb bis über das Kriegsende hinaus im Amt und stand 1943 einem besonderen Kriegskongress vor. Dieser wurde in Wien abgehalten und widmete sich primär den ärztlichen Aufgaben während des Weltkriegs (Forsbach \& Hofer 2015).

\section{Initiativen zur Neukonstituierung}

Formal blieb Hans Eppinger bis zu seinem Suizid am 25. September 1946 Vorsitzender der DGIM. Die Ladung zu einer Zeugenaussage im Nürnberger Ärzteprozess mag zu seiner Selbsttötung beigetragen haben. Er war an der Planung der Meerwasserversuche im Konzentrationslager Dachau beteiligt und hatte seinen Assistenten Wilhelm Beiglböck (1905-1963) für diese Humanexperimente zur Verfügung gestellt. Mehr als vierzig als „Zigeuner" bezeichnete Männer waren zu diesem Zweck aus dem Konzentrationslager Buchenwald nach Dachau gebracht worden. Die meisten von ihnen wurden unter grausamen Umständen gezwungen, teilweise mit $\mathrm{Zu}$ sätzen versehenes Meerwasser zu trinken. $\mathrm{Zu}$ Todesfällen kam es während dieses Humanexperiments nicht. ${ }^{2}$

Durch Eppingers Tod wurde dessen gewählter Stellvertreter, der Würzburger Internist Erich Grafe (1881-1958), Vorsitzender der DGIM. Ursprünglich hätte er dem Wiesbadener Kongress 1942 vorstehen sollen. Auch Grafe hatte sich nicht vom Nationalsozialismus ferngehalten. Ein förderndes Mitglied der SS seit 1933, war er 1934 der SA und 1937 der NSDAP beigetreten. Im Juli 1945 enthob ihn die amerikanische Militärbehörde seines Amtes. Sein Entnazifizierungsverfahren zog sich bis 1948 hin und endete mit der Einstufung in die Gruppe IV (Mitläufer). Darauf erhielt er eine Würzburger Planstelle zurück, wurde aber sofort pensioniert. In einem Rechtsstreit erreichte er 1953 den Status eines Emeritus. In den Nachkriegsjahren leitete er eine Kurklinik, wirkte an der Neubegründung der Deutschen Gesellschaft für Verdauungs- und Stoffwechselkrankheiten 
mit, in der er vor 1945 eng mit Hans Eppinger zusammengearbeitet hatte, und wurde 1954 Vorsitzender der Deutschen Gesellschaft für Ernährung (Jenss \& Lerch 2014: 25).

Im Oktober 1947 fand in Karlsruhe die Regionaltagung der südwestdeutschen Internisten statt. Die dort anwesenden Mitglieder des Vorstands und des Ausschusses, dem zweithöchsten Gremium der DGIM, nutzten die Gelegenheit zu einem Treffen. Die Sitzung fand im Garderobenvorraum des Karlsruher Konzerthauses statt. ${ }^{3}$ In Abwesenheit Grafes wurde sie auf Bitte des Frankfurter Klinikdirektors Franz Volhard (1872-1950) von Ludolph Brauer in seiner inoffiziellen Funktion als Alterspräsident geleitet. ${ }^{4}$ Beide hatten zuvor über Paul Martini in Kontakt mit Arthur Jores (1901-1982) gestanden, der in Hamburg bereits den nordwestdeutschen Nachkriegskongress organisiert hatte. Dabei stand der Gedanke im Raum, den kommenden DGIM-Kongress in Bonn abzuhalten, gegebenenfalls mit Unterstützung durch den benachbarten Kölner Klinikdirektor Hugo Knipping (1895-1984). ${ }^{5}$

Bei der Karlsruher Zusammenkunft der DGIM-Spitze wurde Grafe zwar als amtierender Vorsitzender und damit auch als Präsident eines kommenden Kongresses formal bestätigt. Gleichwohl rechnete man mit seinem baldigen Verzicht und entschloss sich zu einem unkonventionellen, mit Satzung und Geschäftsordnung schwer zu vereinbarenden Schritt: Der bisher nicht im Vorstand vertretene Paul Martini wurde zum „Ersatzmann“ bestimmt. ${ }^{6}$ Er galt fortan „als das am leichtesten aktionsfähige Mitglied des Vorstands" und hatte den in Aussicht genommenen ersten Nachkriegskongress „vorzubereiten und zu leiten, falls Herr Grafe diese Funktion nicht übernehmen kann“.7 Martinis Versicherung, es entspreche „dem Wunsche der großen Mehrzahl der alten Mitglieder" der DGIM, wenn Franz Volhard „bei dieser ersten Tagung, die ja nicht nur eine Nachkriegstagung ist, den Vorsitz" führe, war damit obsolet. ${ }^{8}$ Am 23. Dezember 1947 erklärte Grafe erwartungsgemäß, dem Kongress nicht vorstehen zu wollen. ${ }^{9}$

Die Entscheidung für Martini war so überraschend wie naheliegend. Er hatte im „Dritten Reich“ Abstand zum NS-Regime gehalten, rasch das Vertrauen der amerikanischen Besatzungsmacht gefunden und seit 1932 ohne Unterbrechung der Medizinischen Klinik der Universität Bonn vorgestanden. Den Wiederaufbau von Medizinischer Fakultät und Universität trieb er maßgeblich voran. Hinzu kam sein Wirkungsort Bonn, der allmählich als möglicher Regierungssitz ins Gespräch kam. Schon im Juli 1948 fanden Sitzungen zur Vorbereitung des Verfassungskonvents von Herrenchiemsee im Bonner Museum Koenig statt, am 1. September 1948 nahm dort der Parlamentarische Rat seine Arbeit auf. Dass Paul Martini als Arzt Konrad Adenauers (1876-1967) ein Vertrauter des ersten Kanzlers der Bundesre- 
publik Deutschland werden würde, ließ sich allerdings bei den Karlsruher Beratungen kaum absehen. ${ }^{10}$

Martini nahm die ihm in Karlsruhe zugewiesene Rolle an. Sein Mangel an Begeisterung dürfte jedoch keine Koketterie gewesen sein, hätte er doch sogar dem NS-belasteten Erich Grafe gerne den Vortritt gelassen. An ihn schrieb er am 15. Dezember 1947: „Was mich selbst anlangt, so wäre ich offen gestanden auch aus Egoismus sehr erbaut, wenn dieser Kelch in diesem Jahr an mir vorüber ginge. Ich habe in der Universität das Dekanat und dazu noch eine ganze Menge anderer Ämter am Halse und [bin] so völlig saturiert." ${ }^{11}$ Damit kannte nun auch Grafe aus erster Hand Martinis Haltung. Zuvor hatte der Grandseigneur der DGIM, der 82-jährige Ludolph Brauer, Grafe gegenüber versucht, Martini ins Unrecht zu rücken (Bedenbecker 2014). Grafe aber war umsichtig vorgegangen, zumal er sich der schwierigen rechtlichen Situation bewusst war. Er sah Brauer als seinen Stellvertreter und kommissarischen Vorsitzenden, Martini aber als Leiter des kommenden Kongresses. ${ }^{12}$ Martini legte seine Sicht der Dinge Ende Januar 1948 gegenüber DGIM-Geschäftsführer Friedrich Kauffmann nieder:

Herr Grafe hat mir am 23. Dezember 1947 geschrieben, dass er nicht in der Lage sei, den Kongress zu leiten. Es sei erstens sehr fraglich, ob ein Mitläufer einen allgemeinen Deutschen Kongress leiten könne und weiterhin sei er auch nicht wieder in sein Amt eingesetzt. Er hat mir weiterhin am 2.1. [...] telegraphiert: Mit Karlsruher Tagung unter Ihnen einverstanden. Darüber hinaus haben mich sowohl Grote wie Bürger [...] gebeten, den Kongress vorzubereiten und zu leiten. Danach kann ich mich der Verpflichtung, wenigstens den Kongress vorzubereiten, nicht mehr entziehen. ${ }^{13}$

Zu der schwierigen rechtlichen Basis gehörte die Wahl des DGIM-Vorstands auf der Karlsruher Ausschusssitzung. Gewählt wurden neben Grafe und Martini Louis Grote (1886-1960), Max Bürger (1885-1966), Johann Daniel Achelis (1898-1963) als Kassenführer und als ständiger Geschäftsführer sowie Nachfolger Anton Géronnes traditionell der ärztliche Direktor der medizinischen Abteilung des Krankenhauses Wiesbaden, Friedrich Kauffmann. Aus dem letzten, ursprünglich nur für das Jahr 1940/41 gewählten Vorstand, dem außer Eppinger und Grafe noch Hans Dietlen (1879-1955), Wilhelm Nonnenbruch (1887-1959) und Anton Géronne angehört hatten, war nur Grafe übrig geblieben. ${ }^{14}$ Um eine klare Abgrenzung vom Nationalsozialismus handelte es sich nicht. Auch nach damaligem Kenntnisstand mussten Grafe, Grote und Achelis als belastet gelten.

Louis Ruyter Radcliffe Grote, Sohn eines bei Liverpool geborenen amerikanischen Schmetterlingsforschers, galt als schillernder Internist ohne 
Berührungsängste zu Naturheilkunde und anderen alternativen Behandlungsmethoden. Obwohl selbst kein NSDAP-Mitglied, stand er führenden NS-Ärzten wie Ernst Günther Schenck (1904-1998, seit 1931 DGIM-Mitglied) und Karl Kötschau (1892-1981) mit ihren Vorbehalten gegenüber der „Schulmedizin“ nahe. In dem 1934 in Rudolf-Heß-Krankenhaus und nach dem Englandflug des Namensgebers 1941 sowie Wagners Krebstod 1939 in Gerhard-Wagner-Krankenhaus umbenannten Dresdener Stadtkrankenhaus waren Grote und Alfred Karl Brauchle (1898-1964) die „eigentlichen Repräsentanten des Versuchs, die Wirksamkeit naturheilkundlicher Verfahren in der Klinik zu erproben und damit die erstrebte Verbindung zwischen Schulmedizin und Naturheilkunde zu erreichen" (Heidel 2008: 127). Im Krankenhaus wurde ein erbbiologisch-rassenhygienischer Schwerpunkt eingerichtet, Grote war an Verfahren des Erbgesundheitsobergerichts Sachsen beteiligt und in mindestens sieben Fällen für Zwangssterilisierungen mitverantwortlich. Darüber hinaus agierte Grote zeitweise als Beisitzer am Erbgesundheitsgericht Zwickau (ebd.: 133). In der Nachkriegszeit war Grote zunächst in Wetzlar und ab 1952 als Leiter der Klinik Glotterbad nahe Freiburg ärztlich tätig.

Johann Daniel Achelis war als Ministerialrat im preußischen Kultusministerium 1933/34 „der Architekt der ,Säuberung' der deutschen Hochschulen“ (Heiber 1991: 392). ${ }^{15} 1934$ zum Ordinarius für Physiologie in Heidelberg berufen, entwickelte er großen Ehrgeiz beim Ausbau der dortigen medizingeschichtlichen Lehre. Als Dekan von 1942 bis 1945 hielt er engen Kontakt nach Berlin. Für die Luftwaffe stellte er „Untersuchungen zur Reizphysiologie der Wärmeregulation“ an. 1944 gehörte er zum wissenschaftlichen Beirat des Bevollmächtigten für das Gesundheitswesen, Karl Brandt (Jasch 2005).

\section{Paul Martini als Beobachter der alliierten Politik in Deutschland}

Martinis Weg in die Innere Medizin hatte nach dem Ersten Weltkrieg an der II. Münchener Klinik unter Friedrich von Müller (1858-1941) begonnen, dem in der Zwischenkriegszeit wohl einflussreichsten Internisten im deutschsprachigen Raum. 1928 erlangte er die Position eines Chefarztes am katholischen St. Hedwigs-Krankenhaus in Berlin, wo er seinen eigenen wissenschaftlichen Schwerpunkt, die Methodenlehre therapeutischer Untersuchungen, zu entwickeln begann. ${ }^{16}$ Auf dem Bonner Lehrstuhl brachte ihn sein anhaltendes Drängen auf die Ausgestaltung und Implementierung methodisch valider Kriterien in der Klinik bald in einen Gegensatz zur Neuen Deutsche Heilkunde, die er vehement ablehnte (Forsbach \& Hofer 
2015: 136). In Bonn erarbeitete sich Martini den Ruf eines katholischen Universitätsmediziners, der sich der aggressiven Einflussnahme der Nationalsozialisten an den Hochschulen widersetzte und wissenschaftliche wie persönliche Integrität $\mathrm{zu}$ wahren wusste. Seine mutig vorgebrachte ablehnende Haltung zum NS-Regime sah er allerdings in keinem Gegensatz zur Nutzung von Handlungsspielräumen, die ihm der Krieg eröffnete. 1940 präsentierte er sich als Teil der Funktionselite des Heeres-Sanitätswesens, als Ideengeber und - mit dem obersten „Beratenden Internisten“ der Wehrmacht Kurt Gutzeit (1893-1957) - als Mitinitiator eines militärmedizinischen Projekts, das aus dem Kriegsgeschehen neue Erkenntnis hinsichtlich der Behandlung von Krankheiten versprach. Bedingt durch den Kriegsverlauf konnte das Projekt nur in Teilen verwirklicht werden. ${ }^{17}$

Paul Martini hatte solcherart Distanz zum NS-Regime gehalten, obwohl er mit Leidenschaft als Beratender Internist der Wehrmacht aufgetreten war. Die hierin liegende Problematik hat er später durchaus reflektiert, sie spielte aber in der unmittelbaren Nachkriegszeit keine Rolle. ${ }^{18}$ Mit dem Freiburger Pathologen Franz Büchner (1895-1991) und dem Heidelberger Physiologen Hans Schaefer (1906-2000) kann Martini als Hauptvertreter einer katholisch geprägten Gruppe von Universitätsmedizinern gelten, die in der Nachkriegszeit die Erneuerung einer christlich-abendländischen Geisteshaltung gerade auch in der Medizin forderten. Sie arbeiteten damit de facto Bundeskanzler Konrad Adenauer zu, der in seiner ersten Regierungserklärung 1949 nicht dem Skeptizismus Oswald Spenglers folgte, sondern dem Konstrukt des christlichen Abendlandes eine optimistischzukunftsweisende Strahlkraft mit antikommunistischer Tendenz zuwies. ${ }^{19}$ Martinis "Anstrengungen in der Richtung einer christlichen Erneuerung“ wurden dabei gegen die bereits im April 1945 als „mechanische Bestrafung" kritisierte Vorgehensweise der Alliierten zur juristischen Verfolgung von Nationalsozialisten instrumentalisiert. ${ }^{20}$

Diese hier grundierte Haltung erklärt Martinis nachsichtigen Umgang mit den Zeitgenossen, die in der NS-Zeit größere Schuld als er auf sich geladen hatten. Ein Konglomerat aus oppositionellem Verhalten, soldatischem Pflichtbewusstsein und Katholizismus machte Martini zu einer auch bei den Vertretern der Besatzungsmächte respektierten Persönlichkeit, die sich Gehör zu verschaffen wusste.

Tatsächlich scheute Martini seit dem Ende des NS-Regimes weniger denn je das offene Wort. Der auf einem Vortrag vor dem Deutschen Städtetag in Goslar basierende Aufsatz „Nahrungsbedarf und Unterernährung“ (1947) ist in dieser Hinsicht ein Schlüsseltext. Martini beschrieb nüchtern die aktuelle Not, kritisierte auch die Verantwortlichen auf Seiten der Alliierten, schlug aber zugleich den Bogen zu den tieferen Ursachen des Elends und den nationalsozialistischen Verbrechen. Die Verschränkung von Hun- 
gererfahrungen in den Konzentrationslagern und in der Nachkriegszeit diente erkennbar nicht der Exkulpation des Nationalsozialismus. Hierzu passt, dass Martinis Beitrag in den Stimmen der Zeit veröffentlicht wurde, der jesuitisch geprägten Kulturzeitschrift, die nach jahrelangen Drangsalierungen 1941 verboten worden war und zu deren Redakteuren der wegen seiner Zugehörigkeit zum Kreisauer Kreis hingerichtete Alfred Delp (1907-1945) gehört hatte.

\section{Martinis Kongressvorbereitungen und der „Gegenplan" Ludolph Brauers}

An christlich-ethischen Grundvorstellungen orientierte sich Martini auch bei seinen Überlegungen zur Zusammensetzung der DGIM-Spitze nach 1945. Zu den Kandidaten für die erste Kongresspräsidentschaft nach dem Ende des NS-Systems zählte Louis Grote. Martini betrachtete ihn als Mann „mit verschwommenen medizinischen Ideen“ und als „eine Fahne des Nationalsozialismus“. ${ }^{21}$ In seinen Erinnerungen schreibt Martini:

Als ich aber deshalb nach dem Krieg seine Kandidatur für die Präsidentschaft der Jahrestagung der deutschen Gesellschaft für innere Medizin für indiskutabel erklärte, hörte ich die [...] Entgegnung [...]: „Ja, Herr Martini, wie soll ich denn Nationalsozialist gewesen sein, ich bin doch Freimaurer III. Grades." Ich weiß heute noch nicht, was III. Grades bedeutet, offenbar etwas, wovor er selbst großen Respekt hatte. $^{22}$

Der mit der Freimaurerei wenig vertraute Martini wusste nicht, dass Grote „Meister“ war, in vielen Logen der dritte und höchste Grad. Wenn auch Grote Anhänger der von den Nationalsozialisten abgelehnten Freimaurerei gewesen sein mag, so stand für Martini seine unmittelbare Beteiligung an NS-Unrecht fest. Mit Erich Grafe hingegen wäre Martini als Kongresspräsident „sehr einverstanden gewesen“, doch sprach gegen ihn seine Absetzung durch „die Amerikaner“. ${ }^{23}$ Max Bürger (1885-1966) wiederum galt Martini als „nicht aktionsfähig“, weil er „jetzt in Leipzig in der russischen Zone“ lehrte. ${ }^{24}$ Seine NS-Vergangenheit scheint er deshalb nicht näher betrachtet $\mathrm{zu}$ haben.

Ende 1947 nahm Martini erneut Kontakt zu Brauer, zu Grafe und zu Achelis als ständigem Kassenführer auf. ${ }^{25}$ Seine Ziele fasste er am 29. Dezember 1947 in einem Brief an Achelis zusammen: „Besonders zur Wiederaufnahme des wissenschaftlichen Lebens und unserer wissenschaftlichen Verbindungen mit dem Ausland“ strebe er "einen allgemeinen Kongress“ 
an. ${ }^{26}$ Realistisch schien ihm ein Kongress zu Pfingsten 1948 im vergleichsweise wenig von Kriegszerstörungen betroffenen Karlsruhe, dem Ort der Tagung der südwestdeutschen Internisten von 1947.27 In Martinis Korrespondenz mit Volhard war positiv noch von Bonn, negativ von Wiesbaden, Hamburg und Mainz die Rede gewesen. Selbst Halle war im Gespräch, zumal, so Volhard, „das russische Gesundheitsamt uns sehr unterstützt haben würde“. Dieser Gedanke aber konnte nicht reifen, weil man auf zu viele Mitglieder hätte „verzichten“ müssen, „die die russische Zone nicht mehr betreten können oder vermeiden wollen“ ${ }^{28}$

Nachdem die südwestdeutsche Internistenzusammenkunft in Karlsruhe vom Oktober 1947 gerade mit Blick auf die Rahmenbedingungen sehr positiv bewertet worden war, zog Martini Bonn als Vorschlag für den Ort des 54. DGIM-Kongresses zurück, vor allem wegen mangelnder Unterkunftsmöglichkeiten. ${ }^{29}$ Der nun trotz offenkundiger Schwierigkeiten vehement für Wiesbaden eintretende Brauer traf auf den Widerstand Martinis und Volhards, der die Rollen klar zuwies: „Was wir in Karlsruhe zu erwarten haben, wissen wir; die Stadt wird sich wieder die Beine ausreissen und die Organisation wird meisterhaft sein. [...] Natürlich wollen wir nach Wiesbaden zurückkehren, aber ich meine erst dann, wenn Unterkunft, Verpflegung und Organisation gesichert sind“. ${ }^{30}$ Volhard stärkte Martini in seinen Briefen nun immer wieder den Rücken; dieser antwortete mit spitzen Bemerkungen über Brauer mit seinem „durch die Jahre potenzierten manischen Temperament", bot Volhard aber zugleich immer wieder den DGIM-Vorsitz an. ${ }^{31}$ Volhard lehnte wiederholt ab und stellte in seinem Schreiben am 16. Dezember 1947 klar, Brauer gehöre nicht zum kommissarischen Vorstand und Grafe sei nur „aus Höflichkeit und nur pro Forma zum 1. Vorsitzenden gewählt worden mit der ausdrücklich[en] reservatio mentalis, dass er z.Z. und für den ersten deutschen Kongress noch nicht als Vorsitzender tragbar ist“. Mit der Leitung des Kongresses sei einzig Martini beauftragt - woran Martini durchaus Zweifel hegte und sich ein klareres Votum erhoffte. ${ }^{32}$

Ein vorläufiges Kongressprogramm konnte Martini zu jenem Zeitpunkt bereits benennen. Er schlug als die drei Hauptthemen vegetatives Nervensystem, Blutfarbstoff und Viruskrankheiten vor, zudem die ihm ideal erscheinenden Vortragenden. ${ }^{33}$

Als sich Martini zum Jahreswechsel 1947/48 für einen Karlsruher Kongress einsetzte, war er mit Blick auf die „Formalitäten mit den Besatzungsmächten“ zuversichtlich: „Ich halte dieses Problem für nicht sehr schwierig, wenn es nicht von uns aus von vorn herein tragischer aufgefasst wird als es ist." ${ }^{34}$ Hier irrte Martini. Es zeigte sich bald, wie schwierig das Überwinden der Zonengrenzen innerhalb Deutschlands, aber auch der Außengrenzen für ausländische Kongressteilnehmer war. Zudem erwiesen sich die stren- 
gen Währungsbestimmungen als Hindernis. Hans Volhard, der in Malmö lebende Sohn von Franz Volhard, berichtete, auch für Skandinavier werde eine Reise nach Karlsruhe teuer sein. Erwogen wurde, ausländischen Kongressteilnehmern „Wohnung und Essen“ zu bezahlen. ${ }^{35}$ Über „wertlos werdendes Geld“ verfügte die DGIM zumindest nach Ansicht von Volhard in ausreichendem Maß. ${ }^{36}$ Er schrieb in diesem Sinne nicht nur seinem Sohn, sondern auch dem von den Nationalsozialisten vertriebenen Ernst Wollheim (1900-1981) in Lund. ${ }^{37}$ Bald aber zeigte sich, dass ein solcher Weg nicht praktikabel war. ${ }^{38}$

Neben dem Problem der beschränkten Reisefreiheit hatten sich die Kongressplaner mit Brauers lauter Kritik an dem in Aussicht genommenen Tagungsort Karlsruhe zu befassen. Angesichts des Erfolgs der südwestdeutschen Regionaltagung rechnete man für den DGIM-Kongress mit mindestens 1.500 Teilnehmern. Am 3. Dezember 1947 fasste Martini in einem Brief an Grafe das Ergebnis einer von ihm durchgeführten Umfrage zusammen:

Eine so grosse Teilnehmerzahl wäre [... in Bonn einschl. Beuel, Godesberg, Köln bis Deutz wenn überhaupt, nur in unbefriedigender Weise unterzubringen. Schon bei der letzten hiesigen Chemikertagung, deren Teilnehmerzahl nicht einmal 800 betrug, musste ein grösserer Teil der Besucher in dem rechtsrheinischen Deutz gegenüber Köln in Bunkern übernachten. ${ }^{39}$

Bei einer fast doppelt so hohen Teilnehmerzahl des Internistenkongresses befürchtete Martini eine „Unzufriedenheit, die umso begreiflicher gewesen wäre, als viele Herren die günstigen Karlsruher Verhältnisse schon kennen gelernt haben." 40

Bei Martinis Umfrage entfielen 17 Stimmen auf Karlsruhe. ${ }^{41}$ Gegen Karlsruhe sprachen sich lediglich Brauer, Grafe und „teilweise“ Gustav von Bergmann aus. Damit war Martinis zurückhaltendes Angebot, Bonn stünde nötigenfalls „zur Verfügung [...], wenn nichts Besseres gefunden“ werde, erledigt. ${ }^{42}$ Dennoch agierte Brauer weiter gegen Karlsruhe und auch gegen Martini, den er mit Bonn verknüpfte und somit als gescheitert ansah. Daraufhin sah sich Martini veranlasst, deutlich zu werden. Er stellte klar, dass er ausdrücklich für Karlsruhe eingetreten und unabhängig von der Ortswahl als „Ersatzmann“ für Grafe bestimmt worden sei. ${ }^{43}$ Das hatte ihm zuvor Volhard unzweideutig bestätigt:

Sie sind nun einmal zum Vorsitzenden des nächsten Kongresses gewählt worden, und ich halte es nicht für opportun, Grote oder Grafe bei dem ersten Kongress mit dem Vorsitz zu betrauen, bei dem wir doch grossen Wert auf den interzonalen Charakter legen wollen. Da 
muss der Vorsitzende meiner Meinung nach absolut hasenrein und von unbefleckter Empfängnis sein. ${ }^{44}$

Gleichwohl schien Brauers „Gegenaktion“ zunächst erfolgreich. ${ }^{45}$ Grafe hatte sich nicht an Martini, sondern an Brauer mit der Bitte gewandt, „als komm. 1. Vorsitzender unserer Gesellschaft [...] die Geschäfte weiter zu leiten“" ${ }^{46}$ Dies konnte für Martini nur bedeuten, dass Grafe entweder die Karlsruher Wahlen von Martini als Nachfolger Eppingers im Vorstand und faktischer Vertreter Grafes als Organisator des noch für Bonn geplanten Kongresses nicht anerkannte oder von diesen nichts wusste. ${ }^{47}$ Martini erbat eine schnelle Antwort und ließ seiner Verärgerung über Brauer freien Lauf: „Die Art, wie Herr Brauer mich [...] persönlich in die Debatte gezogen hat, fand ich als umso unerfreulicher, als er durch meine Haltung auf der Ausschusssitzung genau wissen musste, dass er ein falsches Bild über mich verbreitete." ${ }^{48}$ Fortan agierte Grafe nicht mehr offen gegen Martini. Martini wiederum gab zu verstehen, dass er Vorsitz und Präsidentschaft des avisierten Kongresses gerne Grafe überlassen wolle, trug sogar den Gedanken vor, dass beide gemeinsam das Programm entwickelten, „um für alle Fälle gewappnet zu sein". ${ }^{49}$ Grafe aber verzichtete noch vor Weihnachten 1947.

Von diesem Verzicht offenbar noch nicht in Kenntnis gesetzt, ließ Brauer am 9. Januar 1948 eine Stellungnahme drucken, in der er sich nun wortreich für einen nächsten Kongress in Wiesbaden, womöglich erst zu Ostern 1949, einsetzte. Er berichtete von bereits Mitte 1945 aufgenommenen Bemühungen seinerseits, den Wiesbadener Kongress wiederzubeleben, die durch „lebhafte Zustimmungen“ von „etwa 1.400 Mitgliedern“ flankiert worden seien. Zugleich trat Brauer, sich als Stellvertreter Grafes betrachtend, zurück, um Grafe den Vorsitz zu ermöglichen. Martini sei „wohl für Bonn, aber nicht überhaupt als Vorsitzender eines demnächstigen Kongresses in Aussicht genommen“ worden. ${ }^{50}$ Dieser Interpretation hatte Martini wiederholt, unter anderem in einer Ende November verfassten, nicht abgesandten scharfen Replik auf frühere Äußerungen Brauers, widersprochen. ${ }^{51}$

Brauers Macht schwand, doch er sollte auch in den kommenden Wochen die mit Unterstützung Volhards de facto bereits sehr konkret in der Hand Martinis liegende Kongressvorbereitung stören. 


\section{Die Vorbereitung des ersten Nachkriegskongresses: Karlsruhe als Entscheidung gegen Gesamtdeutschland?}

Die internen Auseinandersetzungen standen in einem engen Zusammenhang mit den von den Besatzungsmächten bereiteten Schwierigkeiten. Den in Aussicht genommenen Tagungsort Karlsruhe nutzten in der Sowjetischen Besatzungszone (SBZ) Verantwortliche für die Behauptung, es solle sich dort um einen westdeutschen Kongress lediglich mit Gästen aus der SBZ handeln. Der Vizepräsident der Deutschen Verwaltung für Volksbildung in der SBZ, der spätere Volkskammerabgeordnete Theodor Brugsch, erläuterte seine Einstellung in einem an Ludolph Brauer und Paul Martini gerichtetem Schreiben recht energisch. Brugsch betonte, „daß grundsätzlich der Kongreß für Innere Medizin, der einst von Leyden (Berlin) begründet worden ist, [...] eine gesamtdeutsche Einrichtung sein muß und bleiben muß“. Weiterhin stellte er klar: „Die Ärzte aus der Ostzone, d.h. der sowjetischen Zone können daher keine Gäste sein, sondern gehören dem Kongreß an. Die Genehmigung des Kongresses für Innere Medizin als gesamtdeutsche Einrichtung muß m. E. vom Kontrollrat genehmigt werden". 52

Während Brugsch den Tagungsort Karlsruhe nicht direkt in Frage stellte, erschwerte Brauer durch seine Korrespondenz mit Brugsch von München aus Martinis Arbeit. Nach Brauers Auffassung stand Karlsruhe synonym für eine westdeutsche Medizinertagung, die „nicht das Recht [habe], sich ,als Congress für Innere Medizin' zu bezeichnen. Dieser Titel gebührt einem gesamtdeutschen Congress in Wiesbaden. Demgemäss muss meines Erachtens eine Lizenz für Wiesbaden[,] aber nicht für Karlsruhe gegeben werden." Brauer, der sich „als Senior des Congresses“ sah, erwartete, dass der formal noch als DGIM-Vorsitzender geführte Grafe ein Ansuchen für einen Wiesbadener Kongress beim Alliierten Kontrollrat einreichte: „Damit würden dann selbstverständlich alle Kollegen der Ostzone nicht als Gäste, sondern als Mitglieder erscheinen." ${ }^{53}$

So überging Brauer Martini, den er nicht erwähnte, und Volhard, den er für die im Osten entstandenen Verstimmungen verantwortlich machte. Auf die irritierende Intervention Brauers, von der er Anfang April durch ein Schreiben von Brauer selbst erfuhr, hat Martini offenbar nicht direkt reagiert. ${ }^{54} \mathrm{Zu}$ groß mussten ihm in der heiklen Situation die Meinungsunterschiede erscheinen. Martini wollte einen baldigen regulären gesamtdeutschen DGIM-Kongress in Karlsruhe, Brauer einen zwangsläufig späteren in Wiesbaden. Zudem fehlte Brauer das politische Gespür für den Umgang mit Medizinern, die durch ihr Verhalten in der NS-Zeit belastet waren. Bei aller Korrektheit im Umgang war Martini erkennbar nicht an einer engeren Zusammenarbeit mit Männern wie Grafe und Achelis interessiert. Auch 
mit dem in der SBZ politisch aktiven Brugsch verfuhr Martini nüchtern und vermied eine Solidarisierung in der Art Brauers. ${ }^{55}$

Unabhängig von den Streitigkeiten hatten Martini und vor Ort in Karlsruhe Franz Kienle Anfang 1948 mit der konkreten Planung des Kongresses begonnen. Kienle stellte zwei Stenotypistinnen ein, Martini eine. ${ }^{56}$ Gegenüber Achelis setzte sich Martini mehr als einmal für Kienle und dessen finanzielle "Bewegungsfreiheit" ein, weil er - anders als sein Vorgänger Géronne früher in Wiesbaden - nicht die Hilfe eines städtischen Verkehrsbüros in Anspruch nehmen konnte. ${ }^{57}$ Das Pressereferat in Karlsruhe übernahm Herbert Schwiegk (1906-1988), ein ehemaliges NSDAP- und SA-Mitglied, das mittelbar mit den Dachauer Unterkühlungsversuchung in Berührung gekommen war. ${ }^{58}$ Martini sorgte dafür, dass ihm eine Sekretärin und trotz der hohen Leihgebühren eine Schreibmaschine zur Verfügung gestellt wurde. ${ }^{59}$

$\mathrm{Zu}$ den Kongressvorbereitungen gehörte die Zusammenstellung des Programms. Auch aus diesem geht hervor, dass ein "westdeutscher Kongress“ nicht beabsichtigt war. Mit Referaten wurden Kurt Herzberg (1896-1976, Greifswald), Wolfgang Heubner (1877-1957, Berlin), Karlheinz Tietze (1912-1996, Leipzig), Josef Nöcker (1919-1989, Leipzig) und Kurt Ballowitz (Berlin) in Karlsruhe erwartet. Wie diese bedurften auch Brugsch und der aus dem Westen Berlins stammende Rudolf Birkner (1911-1983) einer Reisegenehmigung. Mit dieser Liste schrieb Martini am 6. April 1948 Karl Linser (1895-1976), dem Präsidenten der Zentralverwaltung der Gesundheitsverwaltung in der SBZ. Er fügte eindringliche Sätze bei:

Es wäre ausserordentlich bedauerlich, wenn durch die derzeitige „verstimmte Lage“ die Vorträge nicht gehalten werden und diese Offenbarung der Gemeinsamkeit aller Deutschen [sic] Aerzte nicht zum Ausdruck käme. Ich bitte Sie deshalb nochmals, sofern dies noch nötig sein sollte, bei der Militär-Regierung in Karlshorst alles zu tun, damit wir unser Ziel erreichen. ${ }^{60}$

Spätestens jetzt, Anfang April 1948, war klar, dass der Schlüssel zu einem nicht nur de jure, sondern auch de facto gesamtdeutschen DGIM-Kongress in der SBZ lag.

Martini konnte sich nicht ausschließlich diesem Problemfeld widmen. Im Bewusstsein der politischen Dimension des Kongresses musste er parallel Zeichen in mehrere Richtungen setzen - gegenüber den Besatzungsmächten, den Mitläufern des Nationalsozialismus, den von den Nationalsozialisten Verfolgten und der internationalen Wissenschaftsgemeinde.

Von den Besatzungsmächten erwartete er die Zulassung eines gesamtdeutschen DGIM-Kongresses, zu dem alle Interessierten aus dem In- und Ausland anreisen konnten. Durch seine demonstrative Freundlichkeit ge- 
genüber Erich Grafe und die Zulassung eines Referenten wie Georg Schaltenbrand (1897-1979) machte er deutlich, dass er deren Wirken in der NS-Zeit als verzeihlich ansah. Schaltenbrand hatte an Multipler Sklerose erkrankten geistig Behinderten experimentell Gehirn-Rückenmark-Flüssigkeit von Affen übertragen (Forsbach \& Hofer 2015: 77).

Martinis Bemühen um eine rasche Wiederanbindung an die international geführten wissenschaftlichen Diskurse fand ihren Ausdruck in seiner Einladungspolitik. So wünschte er sich den Züricher Physiologen Walter Rudolf Hess (1881-1973) als Referenten, dessen Vortrag Martini als „einen keineswegs unwesentlichen Beitrag zu der Aussöhnung der Geister" ansah. ${ }^{61}$ Hess' Teilnahme kam ein besonderer Wert zu, weil andere Kollegen aus dem Ausland die Einladung nach Karlsruhe ausgeschlagen hatten, darunter Cesare Frugoni (1881-1973, Rom), Cecil James Watson (1901-1983, University of Minnesota), J. Erik Jorpes (1894-1973, Stockholm), Paul Govaerts (1889-1960, Brüssel), Gustav Nylin (1892-1961, Stockholm), der allerdings seinen Assistenten Gunnar Biörck (1916-1996) empfahl, Haqvin Malmros (1895-1995, Örebro), Louis Michaud (1880-1956, Lausanne) und Walter L. Palmer (1896-1993, Chicago). ${ }^{62}$ Zudem weigerte sich die J.R. Geigy AG in Basel, das neu entwickelte, unter anderem bei Morbus Parkinson eingesetzte Parpanit auf dem Kongress vorzustellen. ${ }^{63}$ Eingeladen waren neben den Einzelpersonen „sämtliche Direktoren der Schweizer Kliniken und Polikliniken" und die Präsidenten mehrerer schweizerischer Fachgesellschaften - hier mit der Bitte, die Einladung an Interessierte weiterzuleiten. ${ }^{64}$

Ein Stimmungsbericht aus dem Ausland stammt von dem in BerlinCharlottenburg geborenen Hermann Werner Siemens (1891-1969), der 1929 den dermatologischen Lehrstuhl in Leiden eingenommen hatte, dessen Ruf nach Leipzig 1934 von Reichsärzteführer Gerhard Wagner (1888-1939) verhindert worden war und der nach der Besetzung der Niederlande durch die Wehrmacht zeitweilig in Haft geriet (Scholz 1999: 182, 344; Altmeyer 2015). Martini hatte ihm mit der Bitte geschrieben, bei der Suche nach niederländischen Internisten behilflich zu sein, die nach Karlsruhe zu reisen bereit seien. Seine Antwort nahm entsprechende Hoffnungen:

Man fühlt es diesseits eben so, dass es noch zu früh ist. Die Engländer und Amerikaner fühlen es ja sogar so, dass es noch zu früh ist, einem Niederländer die Reise zu seinen deutschen Verwandten zu erlauben. [...] Wir müssen uns darein schicken. Ich finde es ebenso arg wie Sie, würde aber auch nicht gern als einziger Holländer auf einem deutschen Dermatologenkongress paradieren. ${ }^{65}$ 
Martini hatte sich zuvor auch mit Hans Heinrich Berg (1889-1968) in Hamburg beraten, der konkrete Namensvorschläge unterbreitete. ${ }^{66}$ Einladungen gingen schließlich an 21 Ärzte in Schweden, 13 in der Schweiz, je einen in Frankreich und Dänemark, vier in den USA. ${ }^{67}$

$\mathrm{Zu}$ den Eingeladenen zählten Ernst Wollheim und seine Frau in Lund. ${ }^{68}$ Mit Blick auf Wollheim hatte Martini Achelis gebeten, ihn wieder als DGIM-Mitglied zu führen, da er ,ja nur zwangsweise ausgeschieden gewesen" sei. ${ }^{69}$

Für Wollheims Vertreibung trug nicht zuletzt Gustav von Bergmann (1878-1955) Verantwortung. Bergmann war als nüchterner Vertreter des NS-Rechts aufgetreten, der als Prodekan die Berliner Medizinische Fakultät „säuberte“. Noch 1940 befürwortete er die Demontage des Andenkens an frühere Koryphäen des Fachs. Das 1895 errichtete und 1910 vor den Neubau der II. Medizinischen Klinik der Charité platzierte Denkmal für den Mitbegründer der experimentellen Pathologie, Ludwig Traube (1818-1876), wurde im Juli 1940 entfernt. Klinikchef Bergmann ließ dazu die CharitéDirektion wissen, es liege nicht „im Sinne der Weltanschauung des nationalsozialistischen Staates [...], daß das Denkmal eines jüdischen Professors vor der Klinik steht" (Endlich 2008: 248).

1931/32 war der seit 1946 an der Universität München lehrende Bergmann DGIM-Vorsitzender gewesen, hielt sich aber nach dem Ende des NS-Staats bei der Neukonstituierung der Fachgesellschaft zurück. Noch nicht öffentlich artikuliertes und gewiss nicht mehrheitsfähiges Unbehagen aber war vorhanden. In der Rückschau erinnerte sich Kurt Kochsiek, 2010 letzter Träger der anschließend umbenannten Gustav-von-Bergmann-Medaille:

Wir Jüngeren haben uns in den 50-er Jahren gefragt, warum offensichtlich kaum versucht worden ist, wenigstens einige der vertriebenen Professoren zurückzuberufen. Von den Internisten ist lediglich mein Vor-Vorgänger auf dem Würzburger Lehrstuhl 1933, Professor Dr. Ernst Wollheim, aus Schweden zurückberufen worden. Er wurde bereits 1933, wenige Wochen nach der Machtübernahme, als junger Privatdozent der Charité von Professor Dr. Gustav von Bergmann entlassen. ${ }^{70}$

Wenn der Chirurg Rudolf Nissen (1896-1981) in seinen Erinnerungen schreibt, Bergmann sei „stumm“ geblieben, „als seine ,nichtarischen“ Assistenten die Klinik verlassen mussten“, so wird man noch kritischer sagen müssen, dass er aktiv an der Vertreibung beteiligt war (Nissen 1984[1969]: 130; Kinas 2012: 346). Offiziell wurde Wollheim, seit 1929 Privatdozent an der Charité, 1935 nach $\$ 4$ des „Gesetzes zur Wiederherstellung des Berufsbeamtentums“ wegen politischer Unzuverlässigkeit entlassen. Schon 1934 
war er auf eine ordentliche Professur an die Universität Lund (Schweden) gewechselt. Er kehrte 1948 nach Deutschland zurück. Einen Ruf nach Berlin lehnte er ab, nachdem er dort von Theodor Brugsch unfreundlich empfangen worden war und dieser ihn seine vermeintliche neue Arbeitsstätte „nur mit der jüngsten Assistenzärztin der Klinik besichtigen ließ“ (Hubenstorf \& Walther 1994: 84 f.). Daraufhin nahm Wollheim den Ruf auf ein Ordinariat an der Universität Würzburg an (Schagen 2008a: 63).

Bergmann selbst hielt sich seit dem Ende der NS-Zeit innerhalb der DGIM bewusst zurück. Gleichwohl vertrat er gegenüber Martini auf Anfrage klare Standpunkte, hielt Karlsruhe („denkbar unakademischer Ort“) für eine schlechte Wahl, erklärte sich mit Martinis Programm „durchaus einverstanden“ und schimpfte über Brauer, der Bergmann in seine Ränkespiele hatte einbauen wollen: Er sei „senil“, überschütte einen „mit Phrasen“ und nähme nur sich selber wahr. ${ }^{71}$

Nicht nur die Bemühungen um eine Beteiligung von Wissenschaftlern aus dem Ausland stießen auf Schwierigkeiten. Auch die Teilnahme der in der amerikanisch, britisch und vor allem der sowjetisch besetzten Zone lebenden DGIM-Mitglieder blieb ein Problem. Ende 1947 hatte in dieser Angelegenheit Hans Volhard von Frankfurt aus Kontakt mit dem Abteilungsleiter im Office of Military Government for Germany (OMGUS), Hans Simons, aufgenommen. Dieser erklärte die juristische und politische Lage:

Ein Vier-Zonen-Kongress bedarf der Genehmigung des Kontrollrates. Augenblicklich sieht es so aus, als wenn diese Genehmigung kaum gegeben werden würde. [...] Der beste Weg wäre wohl, zu erreichen, dass qualifizierte Persönlichkeiten oder Gruppen in jeder Zone gleichlautende Anträge jede an ihre Militärregierung stellen und Abschriften an die anderen Militärregierungen schicken, so dass von vornherein klar ist, ersten $[\mathrm{s}]$ dass es sich um eine alle Zonen einschliessende deutsche Initiative handelt, zweitens dass alle vier Behörden gleichzeitig und gleichförmig unterrichtet sind. ${ }^{72}$

Ende Januar 1948 war die Situation im Wesentlichen unverändert, wie Volhard Martini nach einem Telefonat mit Simons berichtete. ${ }^{73}$ Über verschiedene Lösungsmodelle wurde diskutiert. Darunter war die von Max Ratschow (1904-1963) unterstützte Idee von Rudolf Cobet (1888-1964), auf der aktuellen Einladung nach Karlsruhe zu vermerken, dass der übernächste „Kongress in der Ostzone stattfinden würde“. ${ }^{74}$ Hoffnung machte diesbezüglich am 10. Februar 1948 ein Schreiben von Karl Linser (1895-1976), dem Präsidenten der Deutschen Zentralverwaltung für das Gesundheitswesen in der SBZ. Er bekundete „lebhaftes Interesse an der Teilnahme der Internisten der Ostzone“. ${ }^{75}$ 
Mitte Februar 1948 übernahm Martini von Volhard die Federführung. Am 17. Februar erinnerte er Lieutenant Colonel Charles Moseley, den Chief Public Health Branch bei der amerikanischen Militärregierung für Hessen in Wiesbaden (OMG Hessen), an dessen zuvor Volhard unterbreitetes Angebot, im Sinne der DGIM bei „Omgus in Berlin zu vermitteln“.76 Er bat, „der Omgus eine Befürwortung zu unterbreiten, damit auch die Internisten und sonstigen Aerzte aus der russischen Zone an dem Kongress teilnehmen können". ${ }^{77}$ In der SBZ selbst sollten Theodor Brugsch (Berlin), Max Bürger (Leipzig) und Walter Brednow (Jena) auf die Militärregierungen einwirken. ${ }^{78}$

Die Bemühungen führten letztlich zum Erfolg. In der Rückschau sah Martini das Problem der Ausreisegenehmigungen aus der Ostzone als zunächst überschätzt an. Es sei „erst schwieriger“ erschienen „als es wirklich war" und betonte, wie leicht es auch im folgenden Jahrzehnt war, dass in der DDR Lehrende wie Max Bürger, Gerhardt Katsch (1887-1961) und Walter Brednow (1896-1976) dem Wiesbadener Kongress vorstehen konnten. ${ }^{79}$

Anders als Vertreter der amerikanischen, britischen und französischen Militärregierung, wurden russische Vertreter nicht ausdrücklich zum Karlsruher Kongress eingeladen. ${ }^{80}$ Eine Ausnahme bildete der Leningrader Kollege Alexei Dmitrijewitsch Speranski (1888-1961), der ein Einladungsschreiben von Paul Martini erhielt. ${ }^{81}$

\section{Der Kongress von 1948: Verantwortung, Schuld und Kontinuitäten}

Während der Vorbereitung des Karlsruher Kongresses scheute Martini weiterhin nicht den Kontakt zu früheren Nationalsozialisten und korrespondierte auch mit Alfred Schittenhelm. Der DGIM-Vorsitzende von 1933 und 1934 war NSDAP-Mitglied seit 1933, unterstützte öffentlich Hitlers Wahl, war zuletzt SS-Standartenführer und galt der Partei noch 1939 als „vollkommen einwandfrei“; erst 1947 wurde er aus der Internierungshaft entlassen (Ratschko 2013: 90). ${ }^{82}$ Martini wählte in seinem Brief Formulierungen, die Schittenhelm mit offensichtlicher Erleichterung aufnahm. Dieser antwortete, „dass unsere Stellung vermutlich gar nicht so sehr stark sich unterschied“. 83

Einige der durch ihr Handeln in der NS-Zeit belasteten Internisten stellten sich auf dem ersten Nachkriegskongress nicht in die erste Reihe und verzichteten auf Vorträge und Referate. An den Diskussionen aber beteiligten sie sich. So meldeten sich nach einem Beitrag des Düsseldorfer Klinikers Kurt Simon über die Enzephalographie unter anderem Karl Oberdisse, Franz Grosse-Brockhoff, Georg Schaltenbrand, Gustav Bodechtel und auch 
Alfred Schittenhelm zu Wort. ${ }^{84}$ Alle hatten der NSDAP angehört und mit Ausnahme von Schaltenbrand, der Obersturmführer des NS-Fliegerkorps war, auch der SS oder SA. ${ }^{85}$ Schaltenbrand, dessen Übertragungen von Affen-Liquor auf geistig behinderte Menschen bekannt waren, zeigte ebenso wenig Scheu auf dem Kongress zu erscheinen wie Schittenhelm, der für die Absetzung und Vertreibung des als Juden verfolgten Vorsitzenden Alfred Lichtwitz und die Selbstgleichschaltung der DGIM mitverantwortlich war. $^{86}$

Von den Genannten waren Bodechtel und Grosse-Brockhoff zudem mit eigenen Beiträgen im Programm aufgeführt. Bodechtel sprach als „Berichterstatter" über das vegetative Nervensystem, Grosse-Brockhoff hielt einen Vortrag zur Pathologie des Blutfarbstoffs. Beide Bereiche zählten neben „Viruskrankheiten“ zu den drei „Hauptverhandlungsthemen“. ${ }^{87}$

Männer wie Schittenhelm pflegten 1948 das Bild des unpolitischen Wissenschaftlers in der Medizin, die keine Hemmungen hatten, von ihren Forschungen und Vorträgen während der NS-Zeit zu berichten. ${ }^{88}$ Es ist bemerkenswert, dass sie sich nicht scheuten, auf dem ersten Nachkriegskongress in Erscheinung zu treten und dass es zugleich keinerlei Maßnahmen gegeben hat, auf ihren Ausschluss hinzuwirken. Gerade im Fall Alfred Schittenhelm war die Symbolik kaum zu verkennen: Die prägende Gestalt der Fachgesellschaft in der NS-Zeit war auch nach 1945 wieder präsent.

Neben Schittenhelm sprach als weiterer DGIM-Vorsitzender der NSZeit Richard Siebeck (1883-1965) in Karlsruhe. Der vergleichsweise zurückhaltende Siebeck war eingeladen, zu Fragen des vegetativen Nervensystems mitzudiskutieren. Er wurde 1933 förderndes Mitglied der SS und trat 1937 der NSDAP bei. Als Dekan der Berliner Medizinischen Fakultät zeigte er gegenüber den nationalsozialistischen Behörden eine gewisse Standhaftigkeit, wenn es um die Förderung von wissenschaftlich fragwürdigen Wehrmachtsangehörigen ging. Wiederholt riet Siebeck Kollegen zu rein wissenschaftlich begründeten Berufungen und führte in einem Schreiben an seinen Wiener Kollegen Eppinger „peinliche Verbeugungen vor anwesenden Zeitgrössen“, wie sie bei Vortragsveranstaltungen üblich geworden seien, als Negativum an. ${ }^{89}$ Innerhalb der DGIM versuchte der Vorstand unter Siebeck eine weitere Politisierung der Kongresse zu verhindern. Nachdem dieser 1936 ganz im Zeichen der Neuen Deutschen Heilkunde gestanden hatte, betonte Siebeck ein Jahr später die Verpflichtung „zu ernster und gediegener Wissenschaft" bei einer Zurückgezogenheit „auf uns selbst“. Es darf als Kritik am Vorjahreskongress und der Politisierung medizinischer Publikationen gewertet werden, wenn Siebeck während der Eröffnung des Kongresses von 1937 erklärte: „Wir wollen nicht mit Redensarten streiten, sondern mit echten Erkenntnissen. [...] Es darf nicht sein, daß aus wissenschaftlichen Anstalten eine Unmenge von Arbeiten 
erscheint, in denen im Grunde kaum etwas von Belang steht, oder Mitteilungen, die keiner Nachprüfung standhalten und die sich in kurzer Frist als wertlos erweisen." ${ }^{90}$

$\mathrm{Zu}$ den politisch klar gegen den Nationalsozialismus positionierten Vortragenden zählten in Karlsruhe Arthur Jores („Vegetatives und endokrines System“) und Wolfgang Heubner („Theoretisches zur Toxikologie des Blutfarbstoffes").

1936 war dem als Privatdozent in Rostock lehrenden Lichtwitz-Schüler Arthur Jores die enge Verbindung zu seinem Lehrer zum Verhängnis geworden. Jores sandte Lichtwitz Sonderdrucke und sein Buch „Grundzüge der inneren Medizin für Zahnärzte und Studierende der Zahnheilkun-

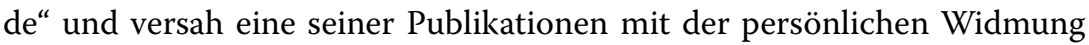
„meinem verehrten Lehrer“ ${ }^{91}$ Eine Denunziation durch seinen Klinikkollegen Werner Böhme machte sein Verhalten öffentlich (Buddrus \& Fritzlar 2007: 76). Jores wurde entlassen und verlor seine venia legendi. Er fand bei der Hamburger „Promonta“, einem Hersteller von Hormonpräparaten, eine Anstellung, die nach einer neuerlichen Denunziation allerdings auch zeitweilig bedroht war. Während des Kriegs tat er als Luftwaffenarzt Dienst und geriet wiederum in Gefahr, weil er wegen Wehrkraftzersetzung angeklagt wurde. Nach dem Ende der NS-Zeit wurde er noch 1945 in HamburgEppendorf Ordinarius, 1950 Rektor der Hamburger Universität und 1969 DGIM-Ehrenmitglied (Danzer 2011: 422; Pongratz 1973: 236-238).

Wolfgang Heubner war ein selbstbewusster „Weltbürger“, der sich nicht scheute, seine abweichende Meinung gegenüber den Nationalsozialisten zu vertreten. Sieht man von einer Mitgliedschaft im Reichsluftschutzbund ab, hielt er sich von nationalsozialistischen Organisationen fern (Kessel 2008: 136; Schagen 2008a: 217). Anders als viele seiner Kollegen versuchte er in der frühen Phase des NS-Regimes, Handlungsspielräume auszuloten. Er riskierte damit sein Berliner Ordinariat und dachte über einen Wechsel zu Ciba nach Basel nach. Auch in der Folgezeit blieb Heubner bei seiner regimekritischen Position. Er verweigerte den Hitler-Gruß und nahm trotz eines ministerialen Verbots an der Trauerfeier des aus „rassischen“ Gründen nach dem „Berufsbeamtengesetz“ entlassenen KWI-Direktors und Nobelpreisträgers Fritz Haber teil (Schagen 2008b: 217-219). Als er 1938 als Gastgeber die Tagung der Deutschen Pharmakologischen Gesellschaft in Berlin eröffnete, huldigte Heubner ganz im Gegensatz zur NS-Ideologie der „weltumspannenden Verbundenheit der Gelehrten, in der die Frage nach Herkunft oder Abkunft gleichgültig ist" (Schagen 2008b: 223). Damit setzte er sich indirekt für den von der Gestapo inhaftierten Grazer Kollegen und Nobelpreisträger Otto Loewi (1873-1961) ein (Kessel 2008: 136). Auch im Hinblick auf dessen spätere Prominenz bemerkenswert ist die Anstellung des Kommunisten Robert Havemann (1910-1982) durch Heubner, den er 
mit Fritz von Bergmann (1907-1982) vor der Vollstreckung des von Roland Freisler (1893-1945) verhängten Todesurteils bewahren konnte (Forsbach \& Hofer 2015: 134f.).

Gleichwohl agierte Heubner an anderen Stellen im Sinne des NS-Systems. Er war einer der beiden Stellvertreter Max Borsts (1869-1946) im Wissenschaftlichen Ausschuss des „Reichsauschusses für Krebsbekämpfung" (Thom 2000: 37). Wohl nicht nur aus taktischen Erwägungen äußerte er sich anlässlich einer USA-Reise, bei der er auch den emigrierten Leopold Lichtwitz besuchte, anerkennend über das „Dritte Reich“ und notierte 1938 in sein Tagebuch, „kein ausgesprochener Gegner des Nationalsozialismus" zu sein (Schagen 2008b: 221f.). Zwar stand er mit seinem Institut nicht an der Spitze der Kriegsforschung, auch nicht der „Kampfstoff-Forschung" (ebd.: 226; Schmaltz 2005). Dennoch gab es Berührungspunkte. Problematisch bleibt zudem seine Beratertätigkeit im Vorfeld der Salzwasserversuche Wilhelm Beiglböcks. ${ }^{92}$

Dies ist die Folie, vor der die öffentliche Auseinandersetzung der DGIMSpitze über die drei Jahre zuvor zu Ende gegangene NS-Diktatur zu sehen ist. Nach Karlsruhe kamen ebenso Symbolfiguren der Anpassung der Inneren Medizin an den Nationalsozialismus wie einstige gegen den NS-Staat auftretende Oppositionelle. Beide Seiten scheinen die gemeinsame Arbeit für den medizinischen Fortschritt im darniederliegenden Deutschland für wichtiger als einen zeichenhaften Boykott des Internistenkongresses angesehen zu haben. Dabei wurde einem einst Verfolgten wie Jores, der auf den Profiteur der Vertreibung seines akademischen Lehrers stieß, deutlich mehr abverlangt als etwa Schittenhelm, der sich an den aktuellen Vorsitzenden Martini recht plump anzubiedern versuchte.

Martini kam bei der Eröffnungsansprache des Kongresses die Aufgabe $\mathrm{zu}$, die DGIM politisch zu positionieren. Er wählte den Weg einer historisch-philosophisch-theologischen Betrachtung und sprach allen Ärzten eine Mitverantwortung für die sprachlich verbrämten NS-Medizinverbrechen zu. Wörtlich heißt es bei Martini:

Autonom gewordenes Fortschrittsstreben [...] durchbrach in der Medizin die Gesetze des ihr spezifischen Objekts, des Menschen, der immer Subjekt bleibt. [...] Die Ärzte, die in Nürnberg auf der Anklagebank saßen, waren zum Teil Verbrecher, mit deren Taten wir nichts zu tun haben wollen. Ein anderer Teil aber ist nichts anders als Fleisch und Geist vom Fleisch und Geist ihrer zeitgenössischen Medizin [...], nicht nur der deutschen [...]. Wer von uns sich von deren Irrwegen ganz freisprechen will, der gleicht den weißgetünchten Gräbern des Evangeliums $\left[{ }^{93}\right]$. Die Hauptursachen des Irrwegs der Medizin unserer Zeit liegen vor uns: Es war die mangelnde Ehrfurcht vor der Schöp- 
fung wie vor ihrem Schöpfer und dazu die Verwischung der Rangordnung der Geschöpfe. Wer eine kontinuierliche Reihe vom Atom bis zum Menschen annimmt, wird, indem er vom Experiment am Leblosen zum Lebendigen und schließlich bis zum Menschen fortschreitet, keine unübersteigbaren Schranken, weil keine grundsätzlichen Unterschiede, finden können.

Konkreter wurde Martini im Rückblick nicht, wandte sich vielmehr, ebenfalls eher allgemein, anderen Themen zu. Mit Sorge erwähnte er die politische Entwicklung in der sowjetischen Besatzungszone, die „Überfüllung des ärztlichen Standes“, den gesunkenen Standard des Medizinstudiums, die Notwendigkeit der „Einordnung der Jungärzte und der ostvertriebenen Kollegen“, die Ernährungslage und die „Lappalisierung“ der Tuberkulosegefahr. Dass der Hunger der Nachkriegszeit auf dem Kongress nicht thematisiert werde, erklärte er mit den bereits erschienenen vielen Publikationen, so dass „neue wissenschaftliche Erkenntnisse“ kaum zu erwarten seien. ${ }^{94}$

Irritierend ist die Lektüre der Totenehrungen in Martinis Eröffnungsrede. In Anwesenheit einiger damals Verantwortlicher, wohl auch Schittenhelms, heißt es passivisch und milde über das Leopold Lichtwitz angetane Unrecht, die Kongressleitung 1933 sei „ihm in allzu großer Nachgiebigkeit vor der neuen Herrschaft dann leider aus der Hand genommen worden“: „Dass er Deutschland verlassen musste, war für ihn ein Schmerz [...], für uns aber ein noch viel größerer Verlust.. ${ }^{\prime 95}$ Hans Eppinger, dem wohl aus Furcht vor dem Nürnberger Ärzteprozess aus dem Leben geschiedenen Kriegsvorsitzenden der DGIM und Mitverantwortlichen von NS-Humanexperimenten, widmete Martini einige, ihn tendenziell entschuldigende Worte mehr: „Die 53. Tagung, die unsere Gesellschaft in Wien 1943 abhalten konnte, verdanken wir seinen Ideen. In tragischer Verkettung, ohne Hoffnung auf eine gerechte Beurteilung seines Denkens, ging er von uns. ${ }^{\text {"96 }}$

Nicht gedacht wurde der von NS-Medizinverbrechen Betroffenen, der Toten und der an Leib und Seele Verletzten.

Trotz der Zurückhaltung im Ton und der Martini eigenen Nachsicht konnte niemand, der seine Eröffnungsrede hörte, die Erinnerung an das vergangene Unrecht verdrängen. Besonders eindrücklich war gegen Ende seiner Ansprache die Begrüßung des auch in der Verantwortung von Gustav von Bergmann vertriebenen, jetzt aus der schwedischen Emigration zurückgekehrten Ernst Wollheim und der Gruß an den in den USA gebliebenen, einst ebenfalls zur Flucht aus Deutschland gezwungenen Siegfried Thannhauser: 
Ich grüße die Zurückgekehrten, in erster Linie unseren Kollegen Wollheim, der unter uns weilt, und nicht weniger diejenigen, die nicht mehr zurückkehren wollen oder können. Dich, Freund Thannhauser, grüße ich besonders herzlich in die Ferne, hier, mitten zwischen Heidelberg und Freiburg, Deinen alten und geliebten Wirkungsstätten. ${ }^{97}$

Im Nachlass Paul Martinis finden sich Texte, die deutlich schärfer auf die NS-Vergangenheit eingehen. In diesen spricht er von „den gräßlichen Erlebnissen des letzten Jahrzehnts“ und von konkreter Schuld: „Deutschland hat sich unter seiner nationalsozialistischen Regierung mit schwerer Schuld beladen. Wir Ärzte schließen uns von der Mitverantwortung für diese Schuld nicht aus. ${ }^{\text {"98 }}$ Auch der Bezug zur politischen Gegenwart ist hier schärfer. Martini verbindet mit der Würdigung fallender Grenzen die klare Markierung des neuen Ost-West-Konflikts und eine klare Positionierung der Ärzteschaft:

Während in der ganzen Welt veraltete Grenzen, Mauern und Vorurteile zwischen den Völkern niedergelegt werden, ist gleichzeitig zu unserer Trauer mitten durch Deutschland eine neue Mauer gewachsen. Aber da der Mensch frei ist und wäre er in Ketten geboren, weil der Geist nicht gezwungen werden kann, wenn er sich nicht zwingen lässt, so können auch wir deutschen Ärzte die geistige Gemeinsamkeit untereinander nur dann verlieren, wenn wir darin einwilligen. ${ }^{99}$

Die DGIM wandte sich unter Martini 1948 nicht nur der NS-Vergangenheit und der weltpolitischen Entwicklung $\mathrm{zu}$, sie forderte angesichts der schwierigen, angeblich nicht korrekt dargestellten Gesundheits- und Ernährungssituation in Deutschland und trotz der Nichtberücksichtigung des Themas auf dem Kongress schnelle Verbesserungen. Martini griff damit seine 1947 auf dem Goslarer Städtetag bereits offensiv angesprochene Hauptsorge wieder auf:

Lebensmittelrationen, die nicht zum Leben ausreichen, bringen in einem industrialisierten und zivilisierten Lande ganz besonders schwere Notstände, da die Möglichkeiten zur Selbsthilfe weitgehend fehlen. Die gesundheitlichen Schädigungen, die so in Deutschland in den letzten Jahren eingetreten sind, sind größer als es offiziell zugegeben wird. [...] Die großen Einbußen an Gesundheit, Arbeitsfähigkeit und Moral, die in Deutschland durch Not und Unterernährung zustande gekommen sind, sind weder vom ärztlichen noch vom menschlichen noch vom wirtschaftlichen Standpunkt aus zu rechtfertigen. ${ }^{100}$

Der Schlusssatz des Entschließungsentwurfs beinhaltet dann doch noch eine exkulpatorische Bemerkung: „Wir bitten die Ärzte der Welt[,] den Ruf 
nach Menschlichkeit über alles und nach einem gesunden Leben für alle zu erheben, den wir selbst unter der Diktatur des Nationalsozialismus nicht erhoben haben, aber auch nicht erheben konnten."101

Letztlich wurden zwei Erklärungen von der Karlsruher Mitgliederversammlung verabschiedet, in die Martinis Überlegungen maßgeblich eingingen. Der Presse wurden diese Erklärungen „a) zur Ernährungslage und b) zur Gesamt-Deutschen Lage“ zugesandt. ${ }^{102}$ Eine Erklärung zur Haltung der DGIM in der NS-Zeit gab es nicht.

Die angesichts der schwierigen Situation verständlichen, rechtlich aber anfechtbaren Personalentscheidungen von Oktober 1947 erforderten Bestätigung oder Revision durch den Kongress. Kassenführer Johann Daniel Achelis war die am stärksten belastete Person an der aktiven DGIM-Spitze. Auch Ludolph Brauer blieb umstritten. Er war zwar kein NSDAP-Mitglied gewesen, hatte aber durchaus mit dem NS-Staat kooperiert. ${ }^{103}$ Martinis Ziel war es, auf dem Kongress sämtliche Gremienmitglieder, die noch als amtierend gelten konnten oder inoffiziell gewählt worden waren, zu versammeln. Dazu zählten die Mitglieder im zweithöchsten Gremium der DGIM, dem Ausschuss. Eine Übersicht zu gewinnen war nicht einfach. Schließlich teilte Achelis Anfang März 1948 Martini eine Liste mit dreizehn „gewählten“ und zwölf „kommissarisch gewählten“ Ausschussmitgliedern mit. ${ }^{104}$ Martini ergänzte sie um „alle früheren Vorsitzenden“, die satzungsgemäß ebenfalls Ausschussmitglieder werden konnten. ${ }^{105}$

Eine auch formal einwandfreie Neuwahl fand am 20. Mai 1948 auf der ordentlichen Mitglieder-Versammlung während des Karlsruher Kongresses statt. Anwesend waren etwa 250 Mitglieder, darunter vom alten Vorstand einzig Erich Grafe. Er hatte an seiner Seite die als „kommissarisch“ bezeichneten Vorstandsmitglieder Paul Martini, Max Bürger und Friedrich Kauffmann als ständigen Schriftführer. ${ }^{106}$ Martini, Bürger und Kauffmann wurden nun „für das Jahr 1948-49“ bestätigt, hinzu gewählt wurden Curt Oehme (1883-1963, Heidelberg) und Walther Frey (1884-1972, Bern). Zu einem „Ersatzmann“ wurde darüber hinaus Philipp Klee (1884-1978, Wuppertal) gewählt. ${ }^{107}$ Keine Rede mehr war von Grote.

Damit blieb der Vorstand hinsichtlich der NS-Vergangenheit seiner Mitglieder gemischt. Von Oehme, seit 1933 förderndes SS-Mitglied und 1941 der NSDAP beigetreten, war bekannt, dass er zwar kriegswichtige Forschung zu Stoffwechsel und Ernährung betrieben hatte, in seiner ärztlichen Praxis freilich durch eine großzügige Versorgung von Kranken mit Hilfe von "Nahrungsmittelattesten“ aufgefallen war. ${ }^{108}$ Der Schweizer Walther Frey hatte früh in Königsberg und Kiel Alfred Schittenhelm kennengelernt. Während der NS-Zeit war er an der Universität Bern tätig (Cottier 1973). Philipp Klee, der enge Mitarbeiter Gerhard Domagks bei der ProntosilForschung für Bayer in Wuppertal-Elberfeld, stand schon aufgrund seiner 
Ehe mit einer aus Ungarn stammenden Jüdin dem NS-Regime ablehnend gegenüber (Grundmann \& Domagk 2001: 49, 166).

Problematisch muss hingegen die Zuwahl von Max Bürger erscheinen, der 1937 von Bonn nach Leipzig gewechselt war. Mochte er nun auch als Vertreter des Ostens gewählt worden sein, er hatte eng mit dem NS-Regime kooperiert. Bürger, der wie Frey unter Schittenhelm in Königsberg und Kiel tätig gewesen war, hatte noch 1933 als Bonner Klinikdirektor das Hissen der NS-Fahne verweigert (Forsbach 2006: 153). Im Vorfeld seines Rufs nach Leipzig schloss er sich der NSDAP und anderen NS-Organisationen an. Während des Zweiten Weltkriegs widmeten sich Bürger und seine Mitarbeiter Forschungen zu Hepatitis und Kreislauf. Insbesondere bei ethisch bedenklichen Unterdruckexperimenten zur Kreislaufforschung ist unklar, wie man an der Leipziger Klinik die Versuchspersonen rekrutierte. Aus den Protokollen geht hervor, dass wenigstens eine Versuchsperson körperlich eingeschränkt war (Thiene 2010: 38). Die unmittelbare Verantwortung für die problematischen Unterdruckversuche in Bürgers Klinik trug sein Assistent Karl Matthes (1905-1962), nach 1945 Ordinarius in Erlangen und Heidelberg. Er war mit einer von den Nationalsozialisten als „Halbjüdin“ klassifizierten Frau verheiratet. Diese Tatsache nutzte Bürger, um Matthes' Anstellung später als Beleg für seine „antifaschistische Einstellung" vorzutragen. ${ }^{109}$

In den Ausschuss der DGIM wurden dreizehn Personen zugewählt, darunter Brugsch, der für konkrete Hilfe für NS-Täter wie Wilhelm Beiglböck bekannte Freiburger Klinikdirektor Ludwig Heilmeyer (1899-1969), der einst verfolgte Arthur Jores und Heinrich Pette (1887-1964), der als NSDAP-Mitglied, Unterzeichner eines Pro-Hitler-Bekenntnisses 1933 und Befürworter einer auf "Auslese gerichteten sozialen Hygiene" in der Neurologie als belastet gelten musste. Pette war zudem stellvertretender „Reichsleiter" der Gesellschaft Deutscher Neurologen und Psychiater hinter Ernst Rüdin gewesen und hatte mit hoher Wahrscheinlichkeit intime Kenntnis von den NS-,Euthanasie“-Verbrechen (Schmuhl 2016: 156-158; Klee 2013: 457).

Organisatorisch versuchte der Karlsruher Kongress trotz der wirtschaftlich angespannten Lage an die Traditionen der Vorkriegszeit anzuknüpfen. Er begann im Vortragssaal der II. Medizinischen Klinik der Städtischen Krankenanstalten mit der Vorstands- und Ausschusssitzung am späten Nachmittag des 17. Mai 1948. Es folgte der „Empfangsabend“ im Studentenhaus der Osthochschule, dem wegen der dortigen Essensausgabe eine zentrale Bedeutung zukam. Am zweiten Abend wurde am Haupttagungsort, dem Städtischen Konzerthaus, „Der Rosenkavalier“ als kultureller Höhepunkt angeboten. ${ }^{110}$ 
Die Essensverpflegung gestaltete sich einen Monat vor der Währungsreform kompliziert. Teilnehmer aus der amerikanischen und englischen Zone mussten „Reisemarken“, die aus der französischen und sowjetischen Zone „Interzonenmarken“ aus ihren Heimatorten vorweisen, mindestens im Wert von 100 Gramm Fleisch und 20 Gramm Fett sowie für „Brot, Nährmittel und Kartoffel nach Bedarf“. Diese Marken wurden gegen „Verpflegungskarten" eingetauscht, die man wiederum an den Essensausgaben im Studentenhaus einlösen konnte. „Eßbesteck und Getränkegläser“ hatte man selbst mitzubringen. ${ }^{111}$

\section{Verspätete Auseinandersetzung mit der NS-Vergangenheit}

Die Neukonstituierung der Deutschen Gesellschaft für Innere Medizin, die im Mai 1948 in den ersten Kongress nach dem Ende des NS-Regimes in Karlsruhe mündete, war von dem Bestreben des Vorsitzenden Paul Martini geprägt, NS-Täter wie Gegner der NSDAP, „Mitläufer“ wie in die Flucht Getriebene, im Ausland wie in den unterschiedlichen Besatzungszonen Deutschlands Lebende zusammenzubringen.

Eine tiefergehende Auseinandersetzung mit der Verantwortung der Internisten für das Unrecht der NS-Zeit wäre unter Martini trotz seiner Großmütigkeit gegenüber früheren Nationalsozialisten denkbar gewesen. Doch an einem solchen Weg bestand kein Interesse. Ein entsprechendes Zeichen nach außen war, dass man keine hohen Ansprüche an die politische Integrität der DGIM-Führungsspitze, nicht einmal der Vorsitzenden stellte. Dem NS-kritischen Nichtparteiangehörigen Martini folgte schon beim zweiten Nachkriegskongress, der traditionsgemäß wieder in Wiesbaden stattfand, mit dem freilich vielfach als integer beschriebenen Curt Oehme ein früheres förderndes Mitglied der SS und ehemaliges NSDAPMitglied als Vorsitzender. Dies war in den folgenden Jahrzehnten nicht die Ausnahme, sondern die Regel.

Flankiert wurde diese Vorgehensweise schon 1949 durch die Verleihung von Ehrenmitgliedschaften an den Profiteur der Vertreibung von Leopold Lichtwitz und den DGIM-Vorsitzenden der Selbstgleichschaltung, Alfred Schittenhelm, sowie an Gustav von Bergmann, der Ernst Wollheim in die Emigration getrieben hatte. Die ersten neuen Ehrenmitglieder waren keine vom NS-Regime Verfolgte, sondern dessen frühere Unterstützer.

Die DGIM der Besatzungszeit vermied einen klaren Bruch mit ihren nationalsozialistischen Mitgliedern. Martinis Weg der Integration von einst Verfolgten wie von Mittätern gelang um den Preis einer für die kommenden Jahrzehnte charakteristischen Geschichtsverdrängung (Roelcke et al. 
2014). Die Beschränkung auf wissenschaftliche Erkenntnis und fachpolitische Interessen ließ es $\mathrm{zu}$, dass sich frühere Nationalsozialisten neben Verfolgten wiederfanden - auf den Kongressen, aber auch auf der Liste der Ehrenmitglieder, wo sich Alfred Schittenhelm, Georg Schaltenbrand und Gustav von Bergmann ebenso finden wie Ernst Wollheim, Siegfried Thannhauser und Walter Seitz (1905-1997), der einzig bekannte Angehörige des Widerstands aus den Reihen der DGIM (Forsbach \& Hofer 2015: 137). Nicht alle vom NS-Staat Vertriebenen hielten dies für zumutbar und zogen es vor, im Ausland zu bleiben.

$\mathrm{Zu}$ den langfristigen Folgen der wenig präzisen Erinnerung an die NSZeit kann gerechnet werden, dass es $1982 \mathrm{zu}$ einer Fälschung kam: Hanns Gotthard Lasch (1925-2009), der damalige Vorsitzende, gab gemeinsam mit dem ständigen Schriftführer Bernhard Schlegel (1913-1987) zum hundertjährigen Geburtstag der DGIM einen fast tausendseitigen Band mit den früheren Reden der Vorsitzenden zur Eröffnung der jährlichen Kongresse heraus (Lasch \& Schlegel 1982). Die Reden aus der NS-Zeit wurden jedoch um Passagen gekürzt, die Nähe zur nationalsozialistischen Ideologie aufwiesen. ${ }^{112}$ Diese nachträglichen Eingriffe in den Text, die mit kleineren Textveränderungen einhergehen, sind für den Leser nicht kenntlich gemacht worden. ${ }^{113}$ Die Fälschung wiegt schwer, weil der Eindruck erweckt wurde, es handele sich um faksimilierte Seiten der originalen Tagungsdokumentationen „Verhandlungen der Deutschen Gesellschaft für Innere Medizin“.

Ein Wandel innerhalb der DGIM wurde zu Beginn des 21. Jahrhunderts erkennbar. Aus den eigenen Reihen wurde die Fälschung aufgedeckt und bekannt gemacht (Broglie \& Schuster 2010). Die Enthüllungen waren Anstoß zu einer breit angelegten Erforschung der Geschichte der Fachgesellschaft seit 1933, die 2015 zunächst in eine Kongressausstellung über die NS-Zeit mündete. Die 2010 letztmalig verliehene Gustav-von-BergmannMedaille, die höchste Auszeichnung der DGIM, trägt seit 2014 den Namen von Leopold Lichtwitz.

\section{Anmerkungen}

1 Schmuhl (2016); Halling et al. (2015); Jenss et al. (2013); Topp (2013); Voswinckel (2012); Sachs et al. (2011); Hewelt (2009).

2 Mitscherlich \& Mielke (1949: 61-63); Peter (2001: 470); Peter (2013: 231-233); Weindling $(2012 ; 2015)$.

3 UB der LMU München, Nachlass (NL) Ludolph Brauer, „Meine Bemühungen und Stellungnahme im Interesse der deutschen Gesellschaft für Innere Medizin und des Kongresses zu Wiesbaden" (09.01.1948). 
4. Medizinhistorisches Institut (MHI) Bonn, NL Paul Martini, Nr. 77, Volhard an Martini, 17.11.1947.

$5 \quad$ UB der LMU München, NL Brauer, Volhard an Brauer, 19.09.1947.

6 MHI Bonn, NL Martini, Nr. 77, Martini an Grafe, 15.12.1947, Durchschlag.

7 MHI Bonn, NL Martini, Nr. 77, Martini an Achelis, 29.12.1947, Durchschlag; ebd., Martini an Grafe, 03.12.1947, Durchschlag.

8 MHI Bonn, NL Martini, Nr. 77, Martini an Volhard, 14.09.1947.

9 MHI Bonn, NL Martini, Nr. 77, Martini an Achelis, 29.12.1947, Durchschlag.

10 Zu Martini vgl. (2006: 139-141).

11 MHI Bonn, NL Martini, Nr. 77, Martini an Grafe, 15.12.1947, Durchschlag.

12 MHI Bonn, NL Martini, Nr. 77, Grafe an Martini, 23.12.1947, Abschrift.

13 MHI Bonn, NL Martini, Nr. 77, Martini an Kauffmann, 29.01.1948, Durchschlag.

14 MHI Bonn, NL Martini, Nr. 77, Martini an Grafe, 03.12.1947, Durchschlag. Ein von Brauer verbreitetes, offenbar verknapptes Protokoll zählt lediglich Grafe, Martini, Bürger, als Kassenführer Achelis und als Geschäftsführer Kauffmann zum Vorstand (MHI Bonn, NL Martini, Nr. 77, Protokoll Ausschusssitzung, 24.10.1947).

15 Heiber 1991: 392. Zu Achelis vgl. auch Flachowsky (2008: 136 f.).

16 Martini, Paul 1932. Methodenlehre der therapeutischen Untersuchung. Berlin. Eine eingehende Auseinandersetzung mit Martini als Methodenreformer der klinisch-therapeutischen Forschung kann im Rahmen dieses Aufsatzes nicht geleistet werden. Diese steht im Mittelpunkt eines aktuell an der Universität Münster durchgeführten DFGProjekts. Vgl. Stoll et al. (2005).

17 Martini, Paul 1940. Über die Möglichkeit des Fortschritts der inneren Medizin im Kriege. In: Münchener Medizinische Wochenschrift (87): 469-474; Gutzeit, Kurt. Aussichten und Durchführung der vergleichenden Therapie im Kriege. BA-MA RH 12-23/1096; Neumann (2005: 105).

18 Vgl. Martini, Paul 1964. Erinnerungen und Erfahrungen. Unveröffentlichte Autobiographie. Bonn: V, 249-251.

19 Vgl. Spengler, Oswald 1918. Der Untergang des Abendlandes. Umrisse einer Morphologie der Weltgeschichte. Erster Band. Gestalt und Wirklichkeit. Wien/Leipzig; Adenauer, Konrad 1949. Regierungserklärung des Bundeskanzlers. 1. Deutscher Bundestag. Stenographisches Protokoll der 5. Sitzung, 20. September 1949 Bonn: 22-30, 30 und für viele Pöpping (2002) und Hürten (1988).

20 MHI Bonn, NL Martini, Nr. 151 Martini an Captain Luebbers/Militärregierung 19.04.1945, Abschrift. Vgl. Forsbach (2006: 664 f.).

21 Martini, Erinnerungen, V, $36 \mathrm{f}$.

22 Martini, Erinnerungen, V, 37.

23 Martini, Erinnerungen, V, 37.

24 Martini, Erinnerungen, V, 37.

25 MHI Bonn, NL Martini, Nr. 77, Martini an Achelis, 29.12.1947, Durchschlag.

26 MHI Bonn, NL Martini, Nr. 77, Martini an Achelis, 29.12.1947, Durchschlag.

27 MHI Bonn, NL Martini, Nr. 77, Martini an Achelis, 29.12.1947, Durchschlag.

28 MHI Bonn, NL Martini, Nr. 77, Volhard an Martini, 19.09.1947; UB der LMU München, NL Brauer, Volhard an Brauer, 19.09.1947

29 MHI Bonn, NL Martini, Nr. 77, Martini an Vorstand und Ausschuss der DGIM, 02.11.1947, Durchschlag.

30 MHI Bonn, NL Martini, Nr. 77, Volhard an Martini, 07.11.1947. 
31 MHI Bonn, NL Martini, Nr. 77, Martini an Volhard, 10.12.1947. Der Wiesbadener Oberbürgermeister sah Wiesbaden erst wieder ab 1949 imstande, den Kongress auszurichten: UB der LMU München, NL Brauer, OB Wiesbaden an Volhard, 24.07.1947, Abschrift.

32 MHI Bonn, NL Martini, Nr. 77, Volhard an Martini, 16.12.1947; ebd., Martini an Volhard, 20.12.1947.

33 MHI Bonn, NL Martini, Nr. 77, Martini an Achelis, 29.12.1947, Durchschlag.

34 MHI Bonn, NL Martini, Nr. 77, Martini an Achelis, 29.12.1947, Durchschlag.

35 MHI Bonn, NL Martini, Nr. 77, Martini an Achelis, 15.02.1948, Durchschlag.

36 MHI Bonn, NL Martini, Nr. 77, Martini an Achelis, 15.02.1948, Durchschlag.

37 MHI Bonn, NL Martini, Nr. 77, Martini an Achelis, 15.02.1948, Durchschlag.

38 MHI Bonn, NL Martini, Nr. 77, Martini an Achelis, 05.03.1948, Durchschlag: „Sehr bedauerlich ist, dass es nicht so einfach sein wird[,] ausländische Gäste notfalls ganz als unsere Gäste aufzunehmen, so wie Herr Volhard sich das gedacht hat. Vielleicht finden die Rechtsgelehrten doch noch einen Ausweg."

39 MHI Bonn, NL Martini, Nr. 77, Martini an Grafe, 03.12.1947. Siehe auch ebd., Martini an Volhard 14.09.1946.

40 MHI Bonn, NL Martini, Nr. 77, Martini an Grafe, 03.12.1947.

41 MHI Bonn, NL Martini, Nr. 77, Martini an Grafe, 03.12.1947.

42 MHI Bonn, NL Martini, Nr. 77, Martini an Volhard, 14.09.1947.

43 MHI Bonn, NL Martini, Nr. 77, Martini an Grafe, 03.12.1947, Durchschlag.

44 MHI Bonn, NL Martini, Nr. 77, Volhard an Martini, 17.11.1947.

45 MHI Bonn, NL Martini, Nr. 77, Martini an Kienle, 28.11.1947.

46 MHI Bonn, NL Martini, Nr. 77, Martini an Grafe, 03.12.1947, Durchschlag.

47 MHI Bonn, NL Martini, Nr. 77, Ergebnisprotokoll Ausschusssitzung, 24.10.1947.

48 MHI Bonn, NL Martini, Nr. 77, Martini an Grafe, 03.12.1947, Durchschlag.

49 MHI Bonn, NL Martini, Nr. 77, Martini an Grafe, 15.12.1947, Durchschlag.

50 UB der LMU München, NL Brauer, „Meine Bemühungen und Stellungnahme im Interesse der deutschen Gesellschaft für Innere Medizin und des Kongresses zu Wiesbaden“ (09.01.1948).

51 MHI Bonn, NL Martini, Nr. 77, Martini an Brauer, 25.11.1947.

52 MHI Bonn, NL Martini, Nr. 77, Brugsch an Brauer, 08.03.1948. Zu Brugsch vgl. Waibel (2011: 56) und seine Autobiographie Theodor Brugsch, Arzt seit fünf Jahrzehnten, Berlin [Ost] (1987).

53 MHI Bonn, NL Martini, Nr. 77, Brauer an Brugsch, 30.03.1948.

54 MHI Bonn, NL Martini, Nr. 77, Brauer an Martini, 31.03.1948, Durchschlag.

55 MHI Bonn, NL Martini, Nr. 77, Martini an Brugsch, 23.03.1948, Durchschlag.

56 MHI Bonn, NL Martini, Nr. 77, Martini an Achelis, 15.02.1948, Durchschlag. Siehe zudem: ebd., Martini an Kienle, 29.11.1947.

57 MHI Bonn, NL Martini, Nr. 77, Martini an Achelis, 03.04.1948, Durchschlag.

58 MHI Bonn, NL Martini, Nr. 77, Martini an Achelis, 03.04.1948, Durchschlag; zu Schwiegks Beteiligung an NS-Verbrechen vgl. Klee (2013: 575).

59 MHI Bonn, NL Martini, Nr. 77, Martini an Achelis, 03.04.1948, Durchschlag.

60 MHI Bonn, NL Martini, Nr. 77, Martini an Linser, 06.04.1948, Durchschlag. Ein Doppel ging gleichzeitig mit separatem Anschreiben an Brugsch.

61 MHI Bonn, NL Martini, Nr. 77, Martini an Hess, 31.05.1948; siehe auch ebd., Redemanuskript Martinis, o.D. [1948]. 
62 MHI Bonn, NL Martini, Nr. 77, Frugoni an Martini, 27.04.1948; MHI Bonn, NL Martini, Nr. 77, Watson an Martini, 30.04.1948; MHI Bonn, NL Martini, Nr. 77, Jorpes an Martini, 23.03.1948; MHI Bonn, NL Martini, Nr. 77, Govaerts an Martini, 12.04.1948; MHI Bonn, NL Martini, Nr. 77, Nylin an Volhard/Frankfurt, 25.02.1948 (weitergeleitet vom Sekretariat Volhard/Frankfurt an Martini, 01.03.1948); MHI Bonn, NL Martini, Nr. 77, Malmros an Martini, 05.04.1948; MHI Bonn, NL Martini, Nr. 77, Michaud an Martini, 18.03.1948; MHI Bonn, NL Martini, Nr. 77, Palmer an Martini, 28.04.1948. MHI Bonn, NL Martini, Nr. 77, Geigy AG an Martini, 26.04.1948.

64 MHI Bonn, NL Martini, Nr. 77, Martini an Oehme, 05.12.1948.

65 MHI Bonn, NL Martini, Nr. 77, Siemens an Martini, 13.02.1948.

66 MHI Bonn, NL Martini, Nr. 77, Berg an Martini, 03.03. und 05.03.1948.

67 MHI Bonn, NL Martini, Nr. 77, Martini an Kienle, 02.03. und 10.03.1948.

68 MHI Bonn, NL Martini, Nr. 77, Martini an Kienle, 02.03.1948,

69 MHI Bonn, NL Martini, Nr. 77, Martini an Achelis, 05.03.1948, Durchschlag.

70 Schreiben Kochsiek an Hofer, 03.05.2012.

71 MHI Bonn, NL Martini, Nr. 77, von Bergmann an Martini, 11.03.1948.

72 MHI Bonn, NL Martini, Nr. 77, Simons/OMGUS an Volhard, 09.12.1947, Abschrift.

73 MHI Bonn, NL Martini, Nr. 77, Volhard an Martini, 20.01.1948.

74 MHI Bonn, NL Martini, Nr. 77, Ratschow an Volhard, 06.02.1948, Abschrift.

75 MHI Bonn, NL Martini, Nr. 77, Linser an Volhard. 10.02.1948, Abschrift.

76 MHI Bonn, NL Martini, Nr. 77, Martini an Moseley, 17.02.1948, Durchschlag. Martini schrieb den Namen Moseleys falsch „Mosley“; zur Stellung Moseleys vgl. University of Wisconsin Digital Collections, Information Bulletin No. 147 (November 1948), Key MG Personnel. URL: images.library.wisc.edu/History/EFacs/GerRecon/omg1948n147/ reference/history.omg1948n147.i0020.pdf (15.07.2015), 31.

77 MHI Bonn, NL Martini, Nr. 77, Martini an Moseley, 17.02.1948, Durchschlag.

78 MHI Bonn, NL Martini, Nr. 77, Martini an Moseley, 17.02.1948, Durchschlag.

79 Martini, Erinnerungen, V, 55.

80 MHI Bonn, NL Martini, Nr. 77, Martini an Oehme, 05.12.1948.

81 MHI Bonn, NL Martini, Nr. 77, Martini an Speranski, 02.03.1948.

82 Bundesarchiv (BA) Berlin, BDC.

83 MHI Bonn, NL Martini, Nr. 77, Schittenhelm an Martini, 11.05.1948.

84 Vgl. Verhandlungen 54 (1949), 189-191.

85 BA Berlin, BDC. Bodechtel: NSDAP-Nr. 4055185, SA-Obersturmführer; Oberdisse: NSDAP-Nr. 4403245, Motor-SA-Scharführer; Grosse-Brockhoff: NSDAP-Nr. 5685132, SA (bis 1935) und HJ-Truppenarzt; Schittenhelm: NSDAP-Nr. 2732711, SS-Nr. 259429 (Sturmbannführer i.S. und SS-Führer z.b.V. RFSS (Sanitätsamt); zu Schaltenbrand vgl. Forsbach \& Hofer (2015: 77).

86 MHI Bonn, NL Martini, Nr. 77, Tagungsprogramm „Tagung der deutschen Gesellschaft für innere Medizin vom 19. bis 21. Mai 1948 in Karlsruhe“; zu Schaltenbrand vgl. Forsbach \& Hofer (2015: 77).

87 MHI Bonn, NL Martini, Nr. 77, Tagungsprogramm „Tagung der deutschen Gesellschaft für innere Medizin vom 19. bis 21. Mai 1948 in Karlsruhe“, 3.

88 Vgl. Schittenhelm in: Verhandlungen 54 (1949), 197.

89 Medizinische Universität Wien, Sammlungen und Geschichte der Medizin, Eppinger, Kongressunterlagen, MUW-AS-004424-0051, Siebeck an Eppinger, 25.06.1944; vgl. unten zur verhinderten Berufung Risaks nach Wien.

90 Verhandlungen 49 (1937), 2; zu Siebecks Haltung und Handeln im Nationalsozialismus und in der Nachkriegszeit vgl. ausführlich Roelcke (2016). 
91 Zit. n. Krebs (1981: 76); vgl. Pongratz (1973: 235); Jores (1935).

92 Vgl. zu Heubner ähnlich Forsbach \& Hofer (2015: 134 f.) und zu seiner Mitverantwortung für die Salzwasserversuche ebd.: 64-66.

93 Mt 23, 27-29: „Ihr Heuchler! Ihr seid wie weiß getünchte Gräber - mit einer sauberen, ordentlichen Außenseite, doch innen voller Gebeine und Schmutz."

94 Martini, Eröffnungsrede, 1-11, 4. Vgl. zu den zahlreichen zeitgenössischen medizinischen Stellungnahmen zur Ernährungslage und den nicht weniger zahlreichen geschichtswissenschaftlichen Lokal- und Regionalstudien zum Thema für das Beispiel Freiburg im Breisgau: Seidler \& Leven (2007: 598-600). Die Hungersnot gab zeitgenössisch auch Anlass zu medizinhistorischen Betrachtungen: Rothschuh (1948).

95 Martini, Eröffnungsrede, 6.

96 Martini, Eröffnungsrede, 7.

97 Martini, Eröffnungsrede, 10.

98 MHI Bonn, NL Martini, Nr. 77, Redemanuskript Martinis, o.D. [1948]; ebd., Entschließungs-Entwurf, o.D. [1948].

99 MHI Bonn, NL Martini, Nr. 77, Redemanuskript Martinis, o.D. [1948].

100 MHI Bonn, NL Martini, Nr. 77, Entschließungs-Entwurf, o.D. [1948].

101 MHI Bonn, NL Martini, Nr. 77, Entschließungs-Entwurf, o.D. [1948].

102 DGIM Wiesbaden, Protokolle der Mitgliederversammlung, Bl. 93.

103 Zu Brauers Wirken bis zum Ersten Weltkrieg: Bedenbecker 2014. Mit Blick auf die NSZeit kritisch: Bussche (1989: 15-17) u.ö.

104 MHI Bonn, NL Martini, Nr. 77, Martini an Achelis, 05.03.1948, Durchschlag.

105 MHI Bonn, NL Martini, Nr. 77, Martini an Achelis, 05.03.1948, Durchschlag.

106 DGIM Wiesbaden, Protokolle der Mitgliederversammlung, Bl. 92.

107 DGIM Wiesbaden, Protokolle der Mitgliederversammlung, Bl. 92.

108 Zur Mitgliedschaft Oehmes: BA Berlin, BDC-Dossier Oehme. Vgl. zu Oehme ausführlich: Eckart et al. (2006: 778-780) (Axel W. Bauer) u.ö.

109 Zit. n. Thiene (2010: 90) (Universitätsarchiv Leipzig, Personalakte 1103, Bl. 162).

110 MHI Bonn, NL Martini, Nr. 77, Tagungsprogramm „Tagung der deutschen Gesellschaft für innere Medizin vom 19. Bis 21. Mai 1948 in Karlsruhe“, 4.

111 MHI Bonn, NL Martini, Nr. 77, Tagungsprogramm „Tagung der deutschen Gesellschaft für innere Medizin vom 19. Bis 21. Mai 1948 in Karlsruhe“, 4.

112 Vgl. für viele die Schittenhelm-Rede 1934 (Lasch \& Schlegel 1982: 513; Verhandlungen 46 (1934): 7 f.); es fehlt u. a.: „Eines der größten Verdienste, die sich unser Führer und der Nationalsozialismus um das Deutsche Volk und darüber hinaus um die Welt erworben haben, ist das große Interesse für alle Vererbungsfragen und das aktive Eintreten für die rassenmäßige Pflege des Volkes."

113 Vgl. für viele die Schittenhelm-Rede 1934, wo der Schlusssatz als Eröffnungserklärung der Tagung falsch wiedergegeben wird (Lasch \& Schlegel 1982: 513; Verhandlungen 46 (1934): 8).

\section{Literatur}

Altmeyer, Peter o.J. Hermann Werner Siemens. URL: http://www.enzyklopaediedermatologie.de/artikel?id=13043 (2.8.2015).

Bedenbecker, Claudia 2014. Ludolph Brauer (1865-1951) als Internist und Wissenschaftsreformer. Werdegang und Anfangsjahre als Ärztlicher Direktor des Allgemeinen Krankenhauses Eppendorf in Hamburg. Diss. med., Hamburg. 
Broglie, Maximilian Guido und Hans-Peter Schuster 2010. Deutsche Gesellschaft für Innere Medizin. Die Reden ihrer Vorsitzenden 1982 bis 2010. Stuttgart: Thieme.

Brugsch, Theodor 1987. Arzt seit fünf Jahrzehnten. Berlin [Ost]: Verlag der Nation.

Buddrus, Michael und Sigrid Fritzlar 2007. Die Professoren der Universität Rostock im Dritten Reich. Ein biographisches Lexikon. München: K. G. Saur.

Bussche, Hendrik van den (Hg.) 1989. Medizinische Wissenschaft im „Dritten Reich“. Kontinuität, Anpassung und Opposition an der Hamburger Medizinischen Fakultät. Berlin/ Hamburg: Reimer.

Cottier, Paul 1973. Walter Frey 1884-1972. Verhandlungen der Schweizerischen Naturforschenden Gesellschaft. Wissenschaftlicher und administrativer Teil (153): 255-258.

Danzer, Gerhard 2011. Wer sind wir? Auf der Suche nach der Formel des Menschen. Anthropologie für das 21. Jahrhundert - Mediziner, Philosophen und ihre Theorien, Ideen und Konzepte. Berlin: Springer.

Deichmann, Ute 1995. Biologen unter Hitler. Porträt einer Wissenschaft im NS-Staat, überarb. u. erw. Ausg. Frankfurt am Main: Campus.

Deichmann, Ute 2001. Flüchten, Mitmachen, Vergessen. Chemiker und Biochemiker in der NS-Zeit. Weinheim: Wiley-VCH.

Eckart, Wolfgang U., Volker Sellin und Eike Wolgast (Hg.) 2006. Die Universität Heidelberg im Nationalsozialismus. Heidelberg: Springer.

Endlich, Stefanie 2008. Distanz und Nähe. Braucht Gedenken sichtbare Zeichen? In: Sabine Schleiermacher und Udo Schagen (Hg.). Die Charité im Dritten Reich. Zur Dienstbarkeit medizinischer Wissenschaft im Nationalsozialismus. Paderborn: Schöningh: 247-253.

Epple, Moritz und Birgit Bergmann 2008. Jüdische Mathematiker in der deutschsprachigen akademischen Kultur. Heidelberg: Springer.

Ferdinand, Ursula, Hans-Peter Kröner und Ioanna Mamali (Hg.) 2013. Medizinische Fakultäten in der deutschen Hochschullandschaft 1925-1950. Heidelberg: Synchron.

Flachowsky, Sören 2008. Von der Notgemeinschaft zum Reichsforschungsrat. Wissenschaftspolitik im Kontext von Autarkie, Aufrüstung und Krieg. Stuttgart: Steiner.

Forsbach, Ralf 2006. Die Medizinische Fakultät der Universität Bonn im „Dritten Reich“. München: Oldenbourg.

Forsbach, Ralf und Hans-Georg Hofer 2015. Die Deutsche Gesellschaft für Innere Medizin in der NS-Zeit. Ausstellung aus Anlass des 121. Kongresses der Deutschen Gesellschaft für Innere Medizin 18.-21. April 2015 in Mannheim. Wiesbaden: Deutsche Gesellschaft für Innere Medizin e. V.

Grundmann, Ekkehard und Gerhard Domagk 2001. Der erste Sieger über die Infektionskrankheiten. Münster: LIT.

Halling, Thorsten, Friedrich H. Moll und Heiner Fangerau (Hg.) 2015. Urologie 1945-1990. Entwicklung und Vernetzung der Medizin in beiden deutschen Staaten. Heidelberg: Springer.

Heiber, Helmut 1991. Universität unterm Hakenkreuz. Teil 1: Der Professor im Dritten Reich. Bilder aus der akademischen Provinz. München: K. G. Saur.

Heidel, Caris-Petra 2008. Schauplatz Sachsen: Vom Propagandazentrum für Rassenhygiene zur Hochburg der Kranken-„Euthanasie“. In: Klaus-Dietmar Henke (Hg.). Schriften des Deutschen Hygiene-Museums Dresden. Bd. 7: Tödliche Medizin im Nationalsozialismus. Von der Rassenhygiene zum Massenmord. Köln: Böhlau: 119-148.

Hewelt, Thorsten 2009. Die Geschichte der Deutschen Gesellschaft für Rheumatologie 1927-2007. Halle: Projekte-Verlag Cornelius.

Hubenstorf, Michael und Peter Th. Walther 1994. Politische Bedingungen und allgemeine Veränderungen des Berliner Wissenschaftsbetriebes 1925-1950. In: Wolfram Fischer, Michael Hubenstorf, Klaus Hierholzer und Rolf Winau (Hg.). Exodus von Wissenschaften aus Berlin. Fragestellungen - Ergebnisse - Desiderate. Entwicklungen vor und nach 1933. Berlin: Gruyter: 5-100.

Hürten, Heinz 1988. „Abendland“ - ein Topos bei Besinnung und Neubeginn. Rottenburger Jahrbuch für Kirchengeschichte (7): 27-31.

Jasch, Hans-Christian 2005. Das preußische Kultusministerium und die „Ausschaltung“von „nichtarischen" und politisch mißliebigen Professoren an der Berliner Universität in den 
Jahren 1933 bis 1934 aufgrund des Gesetzes zur Wiederherstellung des Berufsbeamtentums vom 7. April 1933. URL: www.forhistiur.de/zitat/0508jasch.htm (21.12.2016).

Jenss, Harro, Guido Gerken und Markus M. Lerch 2013. 100 Jahre Deutsche Gesellschaft für Verdauungs- und Stoffwechselkrankheiten. München: Dreesbach.

Jenss, Harro und Markus M. Lerch (im Auftrag der Deutschen Gesellschaft für Gastroenterologie, Verdauung- und Stoffwechselkrankheiten) 2014. Tagungen der Deutschen Gesellschaft für Gastroenterologie, Verdauungs- und Stoffwechselkrankheiten. Die Präsidenten von 1914-2014. Greifswald: Sardellus.

Jores, Arthur 1935. Grundzüge der inneren Medizin für Zahnärzte und Studierende der Zahnheilkunde. Leipzig: Thieme.

Kessel, Nils 2008. Biographie als Disziplinentradition. Von der Idealisierung des Pharmakologen Wolfgang Heubner (1877-1957). Medizin, Gesellschaft und Geschichte (27): $133-160$.

Kinas, Sven 2012. Massenentlassungen und Emigration. In: Michael Grüttner und HeinzElmar Tenorth (Hg.). Geschichte der Universität Unter den Linden. Bd. 2: Die Berliner Universität zwischen den Weltkriegen. Berlin: Akademie: 325-404.

Klee, Ernst 2013. Das Personenlexikon zum Dritten Reich. Wer war was vor und nach 1945. 4. Aufl. Frankfurt am Main: Fischer.

Krebs, Hans 1981. Reminiscences and Reflections. In Collaboration with Anne Martin. Oxford: Clarendon Press.

Lasch, Hanns G. und Bernhard Schlegel 1982. Hundert Jahre Deutsche Gesellschaft für innere Medizin. Die Kongreß-Eröffnungsreden der Vorsitzenden 1882-1982. München: Springer.

Maier, Helmut 2015. Chemiker im „Dritten Reich". Die Deutsche Chemische Gesellschaft und der Verein Deutscher Chemiker im NS-Herrschaftsapparat. Weinheim: Wiley-VCH.

Mitscherlich, Alexander und Fred Mielke 1949. Wissenschaft ohne Menschlichkeit. Medizinische und eugenische Irrwege unter Diktatur, Bürokratie und Krieg. Heidelberg: Lambert Schneider.

Neumann, Alexander 2005. „Arzttum ist immer Kämpfertum“. Die Heeressanitätssinspektion und das Amt "Chef des Wehrmachtssanitätswesens" im Zweiten Weltkrieg (1939-1945). Düsseldorf: Droste.

Nissen, Rudolf 1984 [1969]. Helle Blätter, dunkle Blätter. Erinnerungen eines Chirurgen. Stuttgart: DVA.

Oehler-Klein, Siegrid und Volker Roelcke (Hg.) 2007. Vergangenheitspolitik in der universitären Medizin nach 1945: Institutionelle und individuelle Strategien im Umgang mit dem Nationalsozialismus. Stuttgart: Steiner.

Peter, Jürgen 2001. Unmittelbare Reaktionen auf den Prozess. In: Angelika Ebbinghaus und Klaus Dörner (Hg.): Vernichten und Heilen. Der Nürnberger Ärzteprozess und seine Folgen. Berlin: Aufbau: 452-475.

Peter, Jürgen 2013. Der Nürnberger Ärzteprozess - im Spiegel seiner Aufarbeitung anhand der drei Dokumentensammlungen von Alexander Mitscherlich und Fred Mielke. 3. Aufl. Münster: LIT.

Pöpping, Dagmar 2002. Abendland. Christliche Akademiker und die Utopie der Antimoderne 1900-1945. Berlin: Metropol.

Pongratz, Ludwig J. (Hg.) 1973. Psychotherapie in Selbstdarstellungen. Bern: Huber.

Ratschko, Karl-Werner 2013. Kieler Hochschulmediziner in der Zeit des Nationalsozialismus. Die Medizinische Fakultät der Christian-Albrechts-Universität im „Dritten Reich“. Diss. phil., Kiel: Klartext.

Roelcke, Volker, Sascha Topp und Etienne Lepicard (Hg.) 2014. Silence, Scapegoats, SelfReflection: The Shadow of Nazi Medical Crimes on Medicine and Bioethics. Göttingen: Vandenhoeck \& Ruprecht.

Roelcke, Volker 2016. Forschungsbericht: Richard Siebeck und die Medizin im Nationalsozialismus: Haltung und Handeln bis 1945 und in der Nachkriegszeit. URL: http://www. dgpt.de/fileadmin/download/Geschichte_der_DGPT/Roelcke_2016_Siebeck_25_10_ 2016.pdf (21.12.2016).

Rothschuh, Karl Eduard 1948. Medizingeschichtliches zum Hungerödem. Synopsis (1): $15-22$. 
Sachs, Michael, Heinz-Peter Schmiedebach und Rebecca Schwoch 2011. Deutsche Gesellschaft für Chirurgie 1933-1945 - Die Präsidenten. Heidelberg: Kaden.

Schagen, Udo 2008a. Wer wurde vertrieben? Wie wenig wissen wir? Die Vertreibungen aus der Berliner Medizinischen Fakultät 1933. Ein Überblick. In: Sabine Schleiermacher und Udo Schagen (Hg.). Die Charité im Dritten Reich. Zur Dienstbarkeit medizinischer Wissenschaft im Nationalsozialismus. Paderborn: Schöningh: 51-66.

Schagen, Udo 2008b. Freiheit. Von der Freiheit - und den Spielräumen - der Wissenschaft(ler) im Nationalsozialismus: Wolfgang Heubner und die Pharmakologen der Charité 1933 bis 1945. In: Sabine Schleiermacher und Udo Schagen (Hg.). Die Charité im Dritten Reich. Zur Dienstbarkeit medizinischer Wissenschaft im Nationalsozialismus. Paderborn: Schöningh: 207-228.

Schmaltz, Florian 2005. Kampfstoff-Forschung im Nationalsozialismus. Zur Kooperation von Kaiser-Wilhelm-Instituten, Militär und Industrie. Göttingen: Wallstein.

Schmuhl, Hans-Walter 2016. Die Gesellschaft Deutscher Neurologen und Psychiater im Nationalsozialismus, Berlin: Springer.

Scholz, Albrecht 1999. Geschichte der Dermatologie in Deutschland. Berlin: Springer.

Seidler, Eduard und Karl-Heinz Leven 2007. Die Medizinische Fakultät der Albert-LudwigsUniversität Freiburg im Breisgau. Grundlagen und Entwicklungen. Vollständig überarbeitete und erweiterte Neuauflage. München: Karl Alber.

Stoll, Susanne, Volker Roelcke und Heiner Raspe 2005. Gibt es eine deutsche Vorgeschichte der Evidenz-basierten Medizin? Methodische Standards therapeutischer Forschung im beginnenden 20. Jahrhundert. Deutsche Medizinische Wochenschrift (130): 1781-1784.

Thiene, Hendrik 2010. Max Bürger. Ordinarius für Innere Medizin und Direktor der Leipziger medizinischen Universitätsklinik 1937-1945 und 1947-1957. Diss. med., Leipzig.

Thom, Achim 2000. Der Reichsausschuß für Krebsbekämpfung und seine Wirksamkeit in den Jahren 1930 bis 1945. In: Wolfgang U. Eckart (Hg.). 100 Years of Organized Cancer Research - 100 Jahre organisierte Krebsforschung. Stuttgart: Thieme: 37-42.

Topp, Sascha 2013. Geschichte als Argument in der Nachkriegsmedizin. Formen der Vergegenwärtigung der nationalsozialistischen Euthanasie zwischen Politisierung und Historiographie. Göttingen: Vandenhoeck \& Ruprecht.

Voswinckel, Peter 2012. Die Geschichte der Deutsche Gesellschaft für Hämatologie und Onkologie im Spiegel ihrer Ehrenmitglieder 1937-2012. „Verweigerte Ehre“. Dokumentation zu Hans Hirschfeld. Berlin: Deutsche Gesellschaft für Hämatologie und medizinische Onkologie e. V.

Waibel, Harry 2011. Diener vieler Herren. Ehemalige NS-Funktionäre in der SBZ/DDR. Frankfurt am Main: Lang.

Weindling, Paul 2012. Victims, Witnesses, and the Ethical Legacy of the Nuremberg Medical Trial. In: Kim C. Priemel und Alexa Stiller (Hg.). Reassessing the Nuremberg Military Tribunals: Transitional Justice, Trial Narratives, and Historiography. New York: Berghahn: 74-103.

Weindling, Paul 2015. Victims and Survivors of Nazi Human Experiments: Science and Suffering in the Holocaust. London: Bloomsbury Publishing.

Ralf Forsbach

Institut für Ethik, Geschichte und Theorie der Medizin

Medizinische Fakultät

Universität Münster

Von-Esmarch-Straße 62

48149 Münster

Deutschland

ralf.forsbach@ukmuenster.de 
Hans-Georg Hofer

Institut für Ethik, Geschichte und Theorie der Medizin

Medizinische Fakultät

Universität Münster

Von-Esmarch-Straße 62

48149 Münster

Deutschland

hg.hofer@ukmuenster.de 